\title{
ЭЛЕКТРОТЕХНИКА
}

DOI 10.5862/JEST.219.11

УДК 621.313

M.A. Шакиров

УНИВЕРСАЛЬНАЯ ТЕОРИЯ АВТОТРАНСФОРМАТОРА

\author{
M.A. Shakirov
}

\section{UNIVERSAL THEORY OF AUTOTRANSFORMER}

\begin{abstract}
Разработаны основы новой теории двухобмоточного автотрансформатора с получением универсальной 4Т-образной схемы замещения. Универсализм проявляется в отображении в схеме всех магнитных потоков как в окне, так и в магнитопроводе автотрансформатора. С ее помощью показано, что в магнитопроводе короткозамкнутого автотрансформатора возникают сверхи антипотоки (в сравнении с потоком холостого хода), которые могут существенно превышать соответствУющие потоки в короткозамкнутых трансформаторах
\end{abstract}

АВТОТРАНСФОРМАТОР; ПЕРВИЧНАЯ И ВТОРИЧНАЯ ОБМОТКИ; МАГНИТНЫЙ ПОТОК; КОРОТКОЕ ЗАМЫКАНИЕ; СХЕМА ЗАМЕЩЕНИЯ; ХОЛОСТОЙ ХОД.

The paper presents the foundations of a new theory of the two-winding autotransformer with obtaining the universal T-shaped equivalent circuit. The Universalism of the circuit is manifested in mapping the magnetic fluxes in the window and in the yoke of the autotransformer. This shows that the magnetic circuit of a short-circuited autotransformer experiences super - and antifluxes compared to the fluxes when idling. It is proved that these fluxes can be substantially higher than the respective fluxes in the short-circuited transformers.

AUTOTRANSFORMER; PRIMARY AND SECONDARY WINDINGS; MAGNETIC FLUX; SHORT CIRCUIT; EQUIVALENT CIRCUIT; IDLING.

\section{Введение}

При небольших коэффициентах трансформации напряжений в линиях электропередач, применяемых, в частности, для связи высоковольтных сетей смежных напряжений (например, 110 и 220 кВ или 220 и 500 кВ и т. д.), оказывается экономически более выгодным использовать вместо трансформаторов силовые автотрансформаторы. Объясняется это тем, что при одинаковой проходной мощности автотрансформатор имеет меньшие размеры и более высокий КПД по сравнению с трансформатором.

Вместе с тем автотрансформаторам присущ и ряд недостатков, что может привести к потере их электродинамической устойчивости там, где обычный трансформатор будет более надежным. Для выяснения этих обстоятельств необходимо иметь ясную физическую картину процессов, возникающих в автотрансформаторе в любых условиях его работы, в том числе при коротких замыканиях (К3). Однако до настоящего времени теория автотрансформаторов разработана недостаточно. Как любая несостоявшаяся теория, она характеризуется бессистемностью, что проявляется в следующем:

отсутствии схем замещения автотрансформаторов в основных учебниках по теории электрических машин $[1,2]$; 
несогласованности схемных моделей (если они приводятся): в [3] схема замешения построена относительно тока нагрузки, а в [4] - относительно входного тока автотрансформатора;

отсутствии даже упоминания об автотрансформаторах там, где, казалось бы, они должны быть одной из целей рассмотрения наравне с трансформаторами, так как отличаются от них лишь дополнительными электрическими связями $[5,6]$;

несогласованности в обозначениях чисел витков обмоток и коэффициентов трансформации: каждый из авторов учебников [1-4, 7] использует свои, отличные от других, обозначения этих величин, что наблюдается также в статьях $[8,9]$;

чрезвычайно слабом представлении о физических процессах в короткозамкнутых автотрансформаторах.

К этому следует добавить, что все рассуждения в существующей литературе по автотрансформаторам основаны на представлении о раздельных потоках рассеяния каждой из обмоток, что в действительности не имеет места, о чем предупреждали многие авторы начиная с [10], а также [11, 12].

Между тем в настоящее время все эти трудности могут быть преодолены, так как стало более ясным понимание распределения магнитных потоков в силовых двухобмоточных трансформаторах в любом режиме их работы, в том числе возникновения сверх- и антипотоков в короткозамкнутом трансформаторе [13-15].

Сверхпотоком К3 в какой-либо части магнитопровода называется ее поток при установившемся К3, если он превышает магнитный поток холостого хода (XX) в ней при одном и том же напряжении на зажимах трансформатора в обоих режимах, а антипотоком К3 - если он оказывается направленным встречно потоку XX. Таким образом, при К3 в разных частях магнитопровода одновременно сосуществуют встречно направленные потоки.

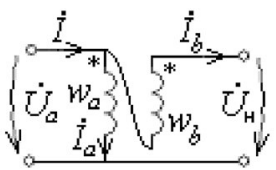

Рис. 1. Электрическая схема повышающего автотрансформатора
Поскольку в автотрансформаторе имеются те же магнитные связи, что и в трансформаторе (рис. 1), то аналогичные потоки при К3 должны, очевидно, возникать и в нем. Причем с большей силой ввиду существенно меньшего его внутреннего сопротивления, т. е. сопротивления K3 ( $\left.z_{\mathrm{K} 3}\right)$.

Цель данной работы - вывод соотношений для определения сверх- и антипотоков в короткозамкнутом автотрансформаторе, что важно для оценки его электродинамической устойчивости в этом состоянии. Достоверное решение этой проблемы возможно только для автотрансформатора со строгим математическим описанием его геометрии. Поэтому здесь, как и в [14, 15], рассматриваются однофазные двухобмоточные броневые автотрансформаторы с концентрическими броневыми ярмами при обычно принимаемом допущении: в пределах обмоток и канала между ними магнитные линии параллельны оси стержня. Это близко к реальности при условии, когда высота обмоток

$$
h_{\text {об }}=h_{\text {оКН }}=h \text {, }
$$

где $h_{\text {окн }}-$ высота окна магнитопровода (рис. 2 , a). Вначале строятся точные схемы замещения для идеализированного автотрансформатора ( $\mu_{\text {сталь }}=\infty$, активные сопротивления обмоток $R_{a}=R_{b}=0$ ), после чего они приспосабливаются для реального автотрансформатора с учетом насыщения. Для определенности рассматривается повышающий автотрансформатор.

\section{Принцип обозначения величин, характеризующих работу автотрансформатора}

Для удобства используется нейтральная по отношению к существующим система обозначений числа витков и коэффициентов трансформации, а также других величин, исключающая путаницу при сравнении и переводе получаемых здесь соотношений на язык обозначений, принятых в различных учебниках. В основу положены индексы $a$ и $b$, совпадающие с обозначениями толщин соответственно внутренней и внешней обмоток (рис. 2). В частности: $w_{a}$ - число витков внутренней обмотки толщиной $a ; w_{a}$ - число витков внешней обмотки толщиной $b$;

$$
n_{b}^{a}=\frac{w_{a}}{w_{b}} ; \quad n_{a}^{b}=\frac{w_{b}}{w_{a}} ; \quad n_{a}=\frac{w_{a}}{w_{a}+w_{b}} ;
$$



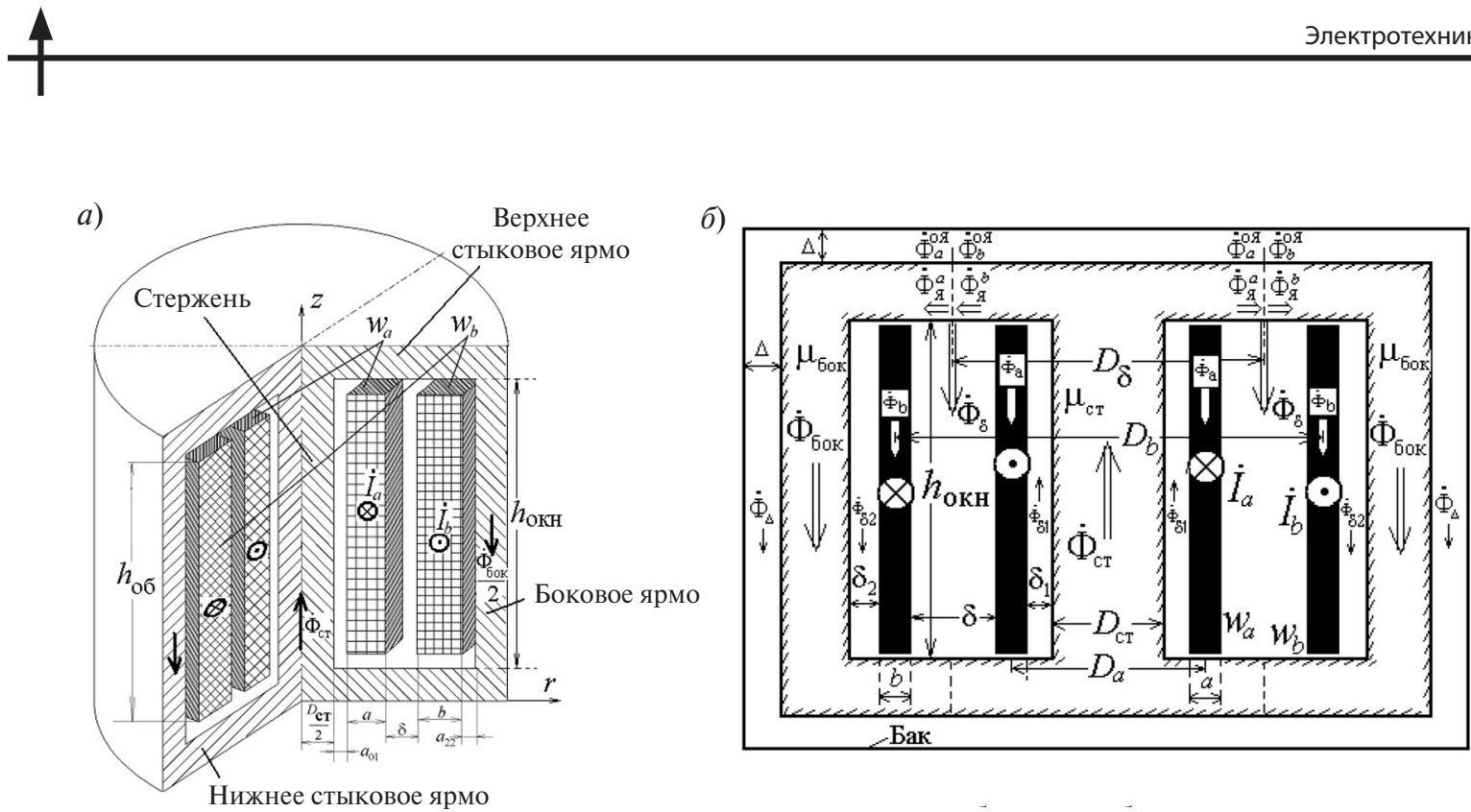

Рис. 2. Магнитная система ( $a$ ) и расположение обмоток в броневом автотрансформаторе с цилиндрическим боковым ярмом (б)

$$
n_{b}=\frac{w_{b}}{w_{a}+w_{b}}
$$

Удобство состоит в том, что здесь нет выделения первичной обмотки, которой обычно присваивается индекс 1. Любая из обмоток далее может рассматриваться как первичная с последующим присвоением ей индекса 1. Из этих соотношений следует:

$$
\begin{gathered}
n_{a}=\frac{1}{1+n_{a}^{b}} ; \quad n_{a}^{b}=\frac{1}{n_{a}}-1 ; \quad n_{b}=\frac{1}{1+n_{b}^{a}} ; \\
n_{b}^{a}=\frac{1}{n_{b}}-1 ; \\
n_{a}=1-n_{b} ; \quad n_{b}=1-n_{a} ; \quad n_{a}^{b} n_{a}=n_{b} ; \\
\\
n_{b}^{a} n_{b}=n_{a} .
\end{gathered}
$$

Аналогичный принцип используется для обозначений магнитных потоков и потокосцеплений:

$\dot{\Phi}_{\text {ст }}-$ магнитный поток в стержне (индекс «ст » от слова «стержень»);

$\dot{\Phi}_{\text {бок }}-$ в боковом ярме (индекс «бок » от слов боковое ярмо);

$\dot{\Phi}_{я}^{a}-$ в стыковом ярме со стороны обмотки $w_{a}$;

$\dot{\Phi}_{\text {я }}^{b}-$ в стыковом ярме со стороны обмотки $w_{b}$

$\dot{\Psi}_{a}$ - потокосцепление обмотки $w_{a}$;

$\dot{\Psi}_{b}-$ потокосцепление обмотки $w_{b}$.
Магнитные потоки в кольцевых каналах окна автотрансформатора снабжены индексами, совпадающими с соответствующей шириной каналов (рис. 2, б):

$\dot{\Phi}_{\delta 1}-$ в канале шириной $\delta_{1}$ между стержнем и внутренней обмоткой;

$\dot{\Phi}_{\delta 2}-$ в канале шириной $\delta_{2}$ между боковым ярмом и внешней обмоткой;

$\dot{\Phi}_{\delta}-$ в канале шириной $\delta$ между обмотками;

$\dot{\Phi}_{a}-$ в толще внутренней обмотки шириной $a$;

$\dot{\Phi}_{b}-$ в толще внешней обмотки шириной $b$.

Очевидно, что сумма этих потоков равна потоку в окне:

$$
\dot{\Phi}_{\mathrm{OKH}}=\dot{\Phi}_{\delta 1}+\dot{\Phi}_{a}+\dot{\Phi}_{\delta}+\dot{\Phi}_{b}+\dot{\Phi}_{\delta 2} .
$$

Магнитные потоки между трансформатором и баком:

$\dot{\Phi}_{a}^{\text {оЯ }}, \dot{\Phi}_{b}^{\text {оЯ }}-$ между баком и соответствующими частями стыковых ярем;

$\dot{\Phi}_{\Delta}-$ в канале между баком и боковым ярMOM.

Магнитные сопротивления кольцевых каналов в окне:

$$
\begin{gathered}
R_{\delta 1}^{\mathrm{M}}=\frac{h}{\mu_{0} s_{\delta 1}} ; \quad R_{\delta 2}^{\mathrm{M}}=\frac{h}{\mu_{0} s_{\delta 2}} ; \quad R_{\delta}^{\mathrm{M}}=\frac{h}{\mu_{0} s_{\delta}} ; \\
R_{a}^{\mathrm{M}}=\frac{h}{\mu_{0} s_{a}} ; \quad R_{b}^{\mathrm{M}}=\frac{h}{\mu_{0} s_{b}},
\end{gathered}
$$

где нижний индекс в обозначении площади $\left(s_{k}\right)$ совпадает с обозначением ширины соответствующего кольцевого канала и его потока (рис. 1, б): 


$$
\begin{gathered}
s_{\delta 1}=\pi\left(D_{\mathrm{cT}}+\delta_{1}\right) \delta_{1} ; \\
s_{a}=s_{1}=\pi D_{a} a ; \\
s_{\delta}=\pi D_{12} a_{12} ; \\
s_{b}=s_{2}=\pi D_{b} b ; \\
s_{\delta 2}=\pi\left(D_{b}+b+\delta_{2}\right) \delta_{2} .
\end{gathered}
$$

Внутренняя индуктивность (индуктивность К3) трансформатора:

$L_{\mathrm{K} 3}^{a}-$ со стороны внутренней обмотки (индуктивность К3, приведенная к обмотке $w_{a}$ );

$L_{\mathrm{K} 3}^{b}$ - со стороны внешней обмотки (индуктивность К3, приведенная к обмотке $w_{b}$ ).

\section{Уравнения для магнитных величин идеализированного автотрансформатора}

По конструкции магнитопровода автотрансформатор тождественен трансформатору при одинаковом расположении обмоток. Поэтому при записи связей между магнитными потоками для автотрансформатора можно воспользовать- ся соотношениями для соответствуюшего трансформатора (рис. 2, б) или его развернутой полной схемой замещения (рис. 3, a), на которой одновременно с электрическими величинами $\left(\dot{I}_{a}, \dot{I}_{b}, \dot{U}_{a}, \dot{U}_{b}\right)$ и индуктивностями $\left(L_{a}, L_{\delta}, L_{b}\right)$ представлены и магнитные величины, т. е. потокосцепления обмоток ( $\left.\dot{\Psi}_{a}, \dot{\Psi}_{b}\right)$, магнитные потоки ( $\dot{\Phi}_{\mathrm{c \tau}}, \dot{\Phi}_{a}, \dot{\Phi}_{\text {я }}^{a}$ и т. д.). Явно локализированы в виде четырех поперечных стрелок потоки в магнитопроводе $\left(\dot{\Phi}_{\text {ст }}, \dot{\Phi}_{я}^{a}, \dot{\Phi}_{я}^{b}, \dot{\Phi}_{\text {бок }}\right)$, что стало основанием в [14] назвать модель (рис. 2, $a$ ) неприведенной 4Т-образной схемой замещения идеализированного трансформатора. Смысл индуктивностей раскрыт в табл. 1, где приведены также соотношения для потоков и потокосцеплений $\Psi_{a}(t), \Psi_{b}(t)$. Эти связи, в отличие от выражений на рис. 3 , представлены для мгновенных значений потоков, что удобно при исследовании переходных процессов.

Левую и правую подсхемы на рис. 3, а разделяет идеальный трансформатор, характеризуемый матричным уравнением
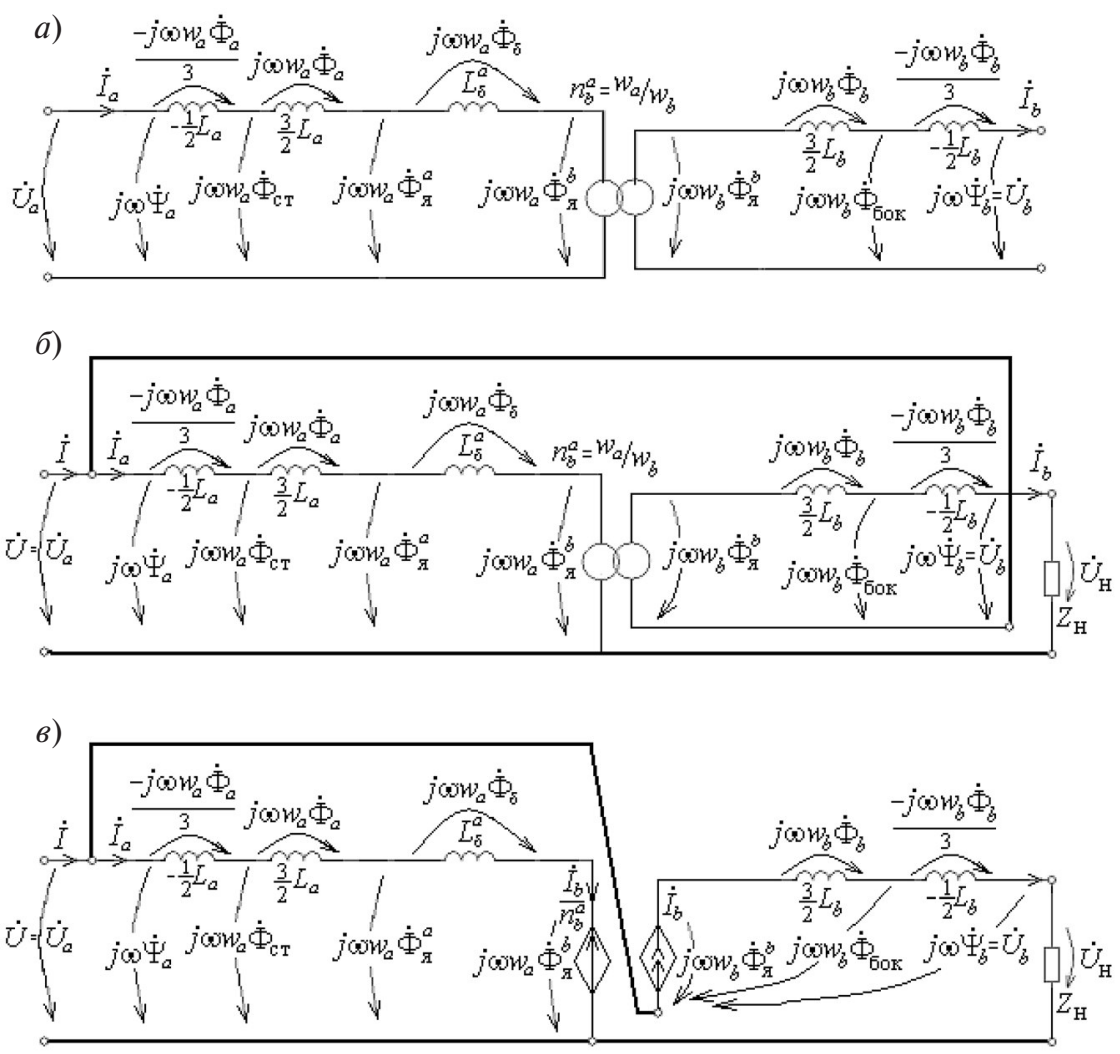

Рис. 3. Неприведенные 4Т-образные схемы замещения идеализированных трансформатора $(a)$ и повышающего автотрансформатора с идеальным элементом $\left(n_{b}^{a}: 1\right)(б)$ и с зависимыми источниками (в) 


\begin{tabular}{|c|c|c|c|}
\hline$j \omega w_{a} \dot{\Phi}_{q}^{b}$ & $n_{b}^{a}$ & & $j \omega w_{b} \dot{\Phi}_{я}^{b}$ \\
\hline$\dot{I}_{a}$ & & $1 / n_{b}^{a}$ & $\dot{I}_{b}$ \\
\hline
\end{tabular}

$=$\begin{tabular}{|c|c|c|}
\hline $1 / n_{a}^{b}$ & & $j \omega w_{b} \dot{\Phi}_{a}^{b}$ \\
\hline & $n_{a}^{b}$ & $\dot{I}_{b}$ \\
\hline
\end{tabular}

где квадратная матрица представляет собой проходную матрицу идеального трансформатора, играющего роль конвертора [16]. Все уравнения для потоков можно получить из схемы рис. $3, a$, используя только второй закон Кирхгофа.

\section{Переход к 4Т-схемной модели} идеализированного автотрансформатора

Для этого достаточно в схеме (рис. $3, a$ ) повторить электрические соединения из схемы рис. 1, что и выполнено на рис. $3, \sigma$ для случая повышающего автотрансформатора с нагрузкой $Z_{\mathrm{H}}$. Полученная таким образом модель является развернутой полной схемой замещении автотрансформатора. Ее также можно назвать неприведенной 4Т-образной схемой замещения идеализированного повышающего автотрансформатора. Для ее расчетов можно перейти к схеме, в кото-

Таблица 1

Соотношения для индуктивностей, потоков и потокосцеплений идеализированного трансформатора $\left(\mu_{\text {сталь }}=\infty\right)$

\begin{tabular}{|c|c|c|c|c|}
\hline $\begin{array}{l}\text { Зона } \\
\text { окна }\end{array}$ & Индуктивность & Поток & $\begin{array}{c}\text { Потокосцепление } \\
\text { обмотки (через элемент } \\
\text { индуктивности) }\end{array}$ & $\begin{array}{c}\text { Потокосцепле- } \\
\text { ние обмотки } \\
\text { (через потоки) }\end{array}$ \\
\hline $\begin{array}{l}\text { Внутрен- } \\
\text { няя } \\
\text { обмотка }\end{array}$ & $\begin{aligned} L_{a} & \approx \frac{w_{a}^{2}}{3 R_{a}^{\mathrm{M}}} \\
L_{a}^{\prime} & \approx \frac{w_{b}^{2}}{3 R_{a}^{\mathrm{M}}} \\
L_{a}^{\prime} & =n_{a}^{b^{2}} L_{a}\end{aligned}$ & $\begin{array}{l}\Phi_{a}(t) \approx \frac{3}{2} \frac{L_{a} i_{a}(t)}{w_{a}} \\
\Phi_{a}(t) \approx \frac{3}{2} \frac{L_{a}^{\prime} i_{b}(t)}{w_{b}}\end{array}$ & $\begin{array}{c}\Psi_{a}(t)= \\
=w_{a} \Phi_{\mathrm{cT}}(t)-\frac{L_{a} i_{a}(t)}{2}\end{array}$ & $\begin{array}{l}w_{a} \Phi_{\mathrm{cT}}(t)- \\
-\frac{w_{a} \Phi_{a}(t)}{3}\end{array}$ \\
\hline $\begin{array}{c}\text { Между } \\
\text { обмотками }\end{array}$ & $\begin{array}{l}L_{\delta}^{a}=\frac{w_{a}^{2}}{R_{\delta}^{\mathrm{M}}} \\
L_{\delta}^{b}=\frac{w_{b}^{2}}{R_{\delta}^{\mathrm{M}}} \\
L_{\delta}^{b}=n_{a}^{b^{2}} L_{\delta}^{a}\end{array}$ & $\begin{array}{l}\Phi_{\delta}(t)=\frac{L_{\delta}^{a} i_{a}(t)}{w_{a}} \\
\Phi_{\delta}(t)=\frac{L_{\delta}^{b} i_{b}(t)}{w_{b}}\end{array}$ & - & $\begin{array}{l}- \\
-\end{array}$ \\
\hline $\begin{array}{l}\text { Внешняя } \\
\text { обмотка }\end{array}$ & $\begin{aligned} L_{b} & \approx \frac{w_{b}^{2}}{3 R_{b}^{\mathrm{M}}} \\
L_{b}^{\prime} & \approx \frac{w_{a}^{2}}{3 R_{b}^{\mathrm{M}}} \\
L_{b}^{\prime} & =n_{b}^{a^{2}} L_{b}\end{aligned}$ & $\begin{array}{l}\Phi_{b}(t) \approx \frac{3}{2} \frac{L_{b} i_{b}(t)}{w_{b}} \\
\Phi_{b}(t) \approx \frac{3}{2} \frac{L_{b}^{\prime} i_{a}(t)}{w_{a}}\end{array}$ & $\begin{array}{c}\Psi_{b}(t)= \\
=w_{b} \Phi_{\text {бок }}(t)+\frac{L_{b} i_{b}(t)}{2}\end{array}$ & $\begin{array}{l}w_{b} \Phi_{\text {бок }}(t)+ \\
+\frac{w_{b} \Phi_{b}(t)}{3}\end{array}$ \\
\hline Окно & $\begin{array}{c}L_{\mathrm{K} 3}^{a}=L_{a}+L_{\delta}^{a}+L_{b}^{\prime} \\
L_{\mathrm{K} 3}^{b}=L_{a}^{\prime}+L_{\delta}^{b}+L_{b} \\
L_{\mathrm{K} 3}^{b}=n_{a}^{b^{2}} L_{\mathrm{K} 3}^{a}\end{array}$ & $\begin{array}{c}\Phi_{\text {окно }}(t) \approx \\
\approx\left(L_{\delta}^{a}+\frac{3}{2}\left(L_{a}+L_{b}^{\prime}\right)\right) \frac{i_{a}(t)}{w_{a}} \\
\Phi_{\text {окно }}(t) \approx \\
\approx\left(L_{\delta}^{b}+\frac{3}{2}\left(L_{a}^{\prime}+L_{b}\right)\right) \frac{i_{b}(t)}{w_{b}}\end{array}$ & - & - \\
\hline
\end{tabular}


рой идеальный трансформатор $\left(n_{b}^{a}: 1\right)$ заменен парой зависимых источников, т. е. источником тока, управляемым током, (ИТУТ)

$$
\dot{I}_{b}=n_{b}^{a} \dot{I}_{a},
$$

и источником напряжения, управляемым напряжением, (ИНУН). Чтобы не вводить новые переменные на схеме, напряжения обозначены через магнитные потоки; поэтому ИНУН представлен уравнением

$$
j \omega w_{a} \dot{\Phi}_{\text {я }}^{b}=n_{b}^{a} j \omega w_{b} \dot{\Phi}_{\text {я }}^{b} .
$$

Входной ток автотрансформатора выражается через токи трансформатора следующим образом:

$$
\dot{I}=\dot{I}_{a}+\dot{I}_{b}=\frac{\dot{I}_{b}}{n_{a}}=\frac{\dot{I}_{a}}{n_{b}} .
$$

Элементы правой части схемы (рис. 3, б) могут быть перенесены через идеальный трансформатор ( $\left.n_{b}^{a}: 1\right)$ в ее левую часть по правилам, изложенным в [17]. В результате получим приведенную 4Т-модель идеализированного повышающего автотрансформатора (см. Приложение).

\section{Свернутые схемы замещения повышающего идеализированного автотрансформатора}

Расчет схем (рис. 3, $a$, б) является тривиальной процедурой. В частности по любой из них можно составить следующую систему уравнений:

$$
\left\{\begin{array}{l}
+j \omega w_{a} \dot{\Phi}_{я}^{b}+j \omega\left(L_{a}+L_{\delta}^{a}\right) \dot{I}_{a}=\dot{U}_{a} \\
-j \omega w_{b} \dot{\Phi}_{я}^{b}+j \omega L_{b} \dot{I}_{b}+\dot{U}_{\mathrm{H}}=\dot{U}_{a} ; \\
\dot{I}_{a}=n_{a}^{b} \dot{I}_{b} .
\end{array}\right.
$$

После подстановки в уравнение (14) правой части (16), умножения его на коэффициент $n_{a}^{b}=w_{b} / w_{a}$ и в результате последующего сложения с уравнением (15) получим выражение

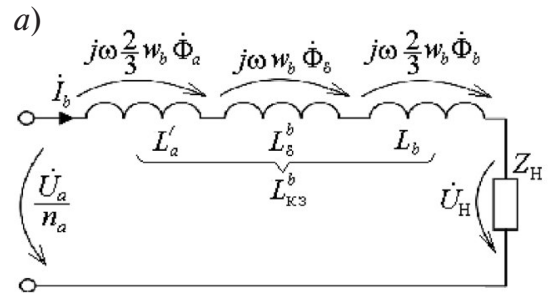

$$
\begin{gathered}
j \omega\left(n_{a}^{b^{2}} L_{a}+n_{a}^{b^{2}} L_{\delta}^{a}+L_{b}\right) \dot{I}_{b}+\dot{U}_{\mathrm{H}}= \\
=\left(1+n_{a}^{b}\right) \dot{U}_{a} \rightarrow \frac{\dot{U}_{a}}{n_{a}},
\end{gathered}
$$

которое с учетом соотношений табл. 1 может быть переписано в виде

$$
j \omega\left(L_{a}^{\prime}+L_{\delta}^{b}+L_{b}\right) \dot{I}_{b}+\dot{U}_{\mathrm{H}}=\frac{\dot{U}_{a}}{n_{a}},
$$

или

$$
j \omega L_{\kappa 3}^{b} \dot{I}_{b}+\dot{U}_{\mathrm{H}}=\frac{\dot{U}_{a}}{n_{a}} .
$$

Этому результату можно поставить в соответствие схему замещения (рис. $4, a$ ), согласующуюся со схемой, приведенной в [3, с. 131]. Из нее следует

$$
\dot{I}_{b}=\frac{\dot{U}_{a}}{n_{a}\left(j \omega L_{\mathrm{K} 3}^{b}+Z_{\mathrm{H}}\right)} .
$$

Подставив $\dot{I}_{b}$ в уравнение (15), нетрудно получить поток

$$
\dot{\Phi}_{\Omega}^{b}=\left(\frac{j \omega L_{b}+Z_{\mathrm{H}}}{n_{a}\left(j \omega L_{\mathrm{K} 3}^{b}+Z_{\mathrm{H}}\right)}-1\right) \frac{\dot{U}_{a}}{j \omega w_{b}} .
$$

Если после подстановки $\dot{I}_{b}=n_{b}^{a} \dot{I}_{a}$ в уравнение (15) и умножения этого уравнения на $n_{b}^{a}=w_{a} / w_{b}$ сложить его с (14), то получим выражение

$$
\begin{gathered}
j \omega\left(L_{a}+L_{\delta}^{a}+n_{b}^{a^{2}} L_{b}\right) \dot{I}_{a}+n_{b}^{a} \dot{U}_{\mathrm{H}}= \\
=\left(1+n_{b}^{a}\right) \dot{U}_{a} \rightarrow \frac{\dot{U}_{a}}{n_{b}},
\end{gathered}
$$

которое с учетом соотношений табл. 1 может быть переписано в виде

$$
j \omega\left(L_{a}+L_{\delta}^{a}+L_{b}^{\prime}\right) \dot{I}_{a}+\dot{U}_{\mathrm{H}}^{a}=\frac{\dot{U}_{a}}{n_{b}},
$$

б)

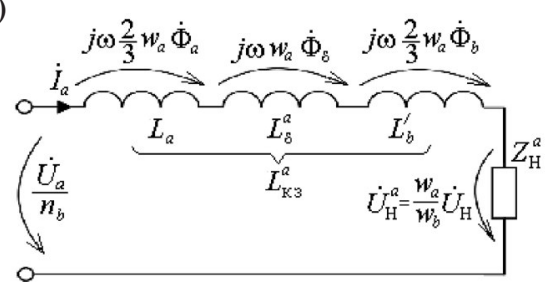

Рис. 4. Упрощенная схема замещения повышающего автотрансформатора относительно тока $\dot{I}_{b}(a)$ и тока $\dot{I}_{a}($ б) 
или

$$
j \omega L_{\mathrm{K} 3}^{a} \dot{I}_{a}+\dot{U}_{\mathrm{H}}^{a}=\frac{\dot{U}_{a}}{n_{b}},
$$

где $\dot{U}_{\mathrm{H}}^{a}-$ напряжение нагрузки, приведенное к внутренней обмотке,

$$
\dot{U}_{\mathrm{H}}^{a}=n_{b}^{a} \dot{U}_{\mathrm{H}}=n_{b}^{a} Z_{\mathrm{H}} \dot{I}_{b}=n_{b}^{a} Z_{\mathrm{H}}\left(n_{b}^{a} \dot{I}_{a}\right)=Z_{\mathrm{H}}^{a} \dot{I}_{a} .
$$

Здесь введено сопротивление нагрузки, приведенное к внутренней обмотке:

$$
Z_{\mathrm{H}}^{a}=n_{b}^{a^{2}} Z_{\mathrm{H}} \text {. }
$$

Этому результату соответствует схема замещения (рис. 4, б). Из нее следует

$$
\dot{I}_{a}=\frac{\dot{U}_{a}}{n_{b}\left(j \omega L_{\mathrm{K} 3}^{a}+Z_{\mathrm{H}}^{a}\right)} .
$$

Подставив $\dot{I}_{a}$ в уравнение(14), получаем поток в виде

$$
\dot{\Phi}_{\text {Я }}^{b}=\left(1-\frac{j \omega L_{a}+j \omega L_{\delta}^{a}}{n_{b}\left(j \omega L_{\mathrm{K} 3}^{a}+Z_{\mathrm{H}}^{a}\right)}\right) \frac{\dot{U}_{a}}{j \omega w_{a}} .
$$

Подставив в выражение $\dot{I}=\dot{I}_{a}+\dot{I}_{b}$ правые части из соотношений для $\dot{I}_{a}$ и $\dot{I}_{b}$, в результате несложных преобразований получаем

$$
\dot{I}=\frac{\dot{U}_{a}}{n_{a}^{2}\left(j \omega L_{\mathrm{K} 3}^{b}+Z_{\mathrm{H}}\right)},
$$

или

$$
\dot{I}=\frac{\dot{U}_{a}}{n_{b}^{2}\left(j \omega L_{\mathrm{K} 3}^{a}+Z_{\mathrm{H}}^{a}\right)} .
$$

Первому из этих соотношений соответствует схема замещения (рис. 5, $a$ ), а второму - схема (рис. 5, б).

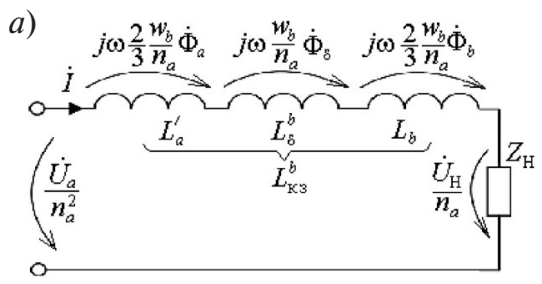

Рис. 5. Упрощенная схема замещения повышающего автотрансформатора

относительно тока $\dot{I}$ с индуктивностью К3 $L_{\text {Кз }}^{b}(a)$ и индуктивностью К3 $L_{\text {кз }}^{a}(б)$ б)

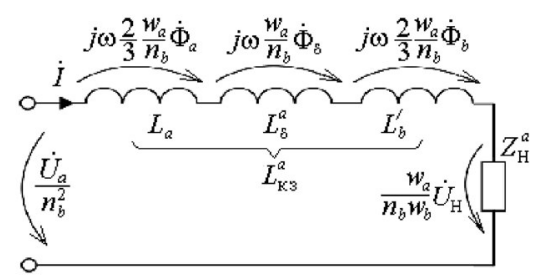

Используя найденные по этим схемам величины, далее по схемам (рис. 3,6, в) можно найти все потоки в стали и окне автотрансформатора. Например, для потока в толще обмотки $w_{a}$ имеем

$$
j \omega w_{a} \dot{\Phi}_{a}=j \omega \frac{3}{2} L_{a} \dot{I}_{a},
$$

откуда

$$
\begin{aligned}
& \dot{\Phi}_{a}=\frac{3}{2} L_{a} \frac{\dot{I}_{a}}{w_{a}}=\frac{3}{2} L_{a} \frac{1}{w_{a}} \dot{I}_{a}= \\
& =\frac{3}{2} L_{a} \frac{1}{w_{a}} \frac{\dot{U}_{a}}{n_{b}\left(j \omega L_{\mathrm{K} 3}^{a}+Z_{\mathrm{H}}^{a}\right)}
\end{aligned}
$$

\section{Родственно эквивалентные схемы замещения автотрансформатора}

Представленные свернутые четыре схемы замещения (рис. 4 и 5) могут быть получены одна из другой в силу того, что, если известна одна из них, то ей можно поставить в соответствие бесчисленное множество «родственных» эквивалентных схем. Это утверждение можно представить в виде следующей леммы.

Лемма. Пусть в качестве исходной задана схема (рис. 6, a), в которой при действии на входе напряжения $\dot{U}$ протекают токи $\dot{I}_{m}, \dot{I}_{n}, \dot{I}_{0}$ и сопротивление $Z_{\mathrm{H}}$ находится под напряжением $\dot{U}_{\mathrm{H}}$. Выберем произвольные два числа $\eta$ и $\xi$, не равные нулю. Назовем родственной схему, в которой приложенное напряжение больше в $\eta$ раз, а все сопротивления увеличены в $\xi$ раз (рис. 6,6 ). В родственной схеме все напряжения увеличатся в $\eta$ раз, все токи - в $(\eta / \xi)$ раз, а все мощности - в $\eta^{2} / \xi$ раз. Родственная схема эквивалентна исходной в том смысле, что по ней можно простым алгебраическим пересчетом найти все токи, напряжения и мощности исходной схемы. 
a)

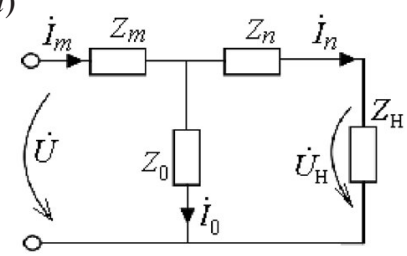

б)

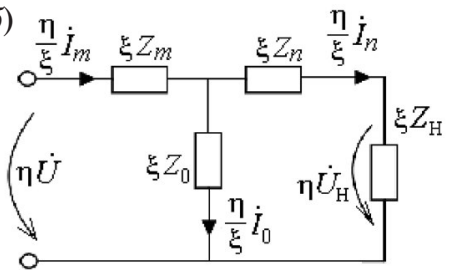

Рис. 6. Преобразование схемы $(a)$ в родственную эквивалентную схему (б)

Все схемы на рис. 4 и рис. 5 родственно эквивалентны. Чтобы показать это, выберем в качестве исходной схему на рис. $4, a$. Коэффициенты $\eta$ и $\xi$, превращающие ее в остальные схемы, представлены в табл. 2. Исходной схеме соответствуют, очевидно, коэффициенты $\eta=1$ и $\xi=1$.

Таблица 2

\section{Синтез родственно эквивалентных схем замещения} идеализированного автотрансформатора

\begin{tabular}{|c|c|c|}
\hline$\eta$ & $\xi$ & $\begin{array}{c}\text { Родственная схема } \\
\text { замещения }\end{array}$ \\
\hline 1 & 1 & Рис. 4, $a$ \\
$n_{b}^{a}$ & $n_{b}^{a^{2}}$ & Рис. 4, $\sigma$ \\
$1 / n_{a}$ & 1 & Рис. 5, $a$ \\
$n_{b}^{a} / n_{b}$ & $n_{b}^{a^{2}}$ & Рис. 5, $\sigma$ \\
$n_{a}$ & $n_{a}^{2}$ & Рис. 7, $a$ \\
\hline
\end{tabular}

На рис. 7, $a$ синтезирована схема, в которой входные напряжение и ток совпадают с соответствующими величинами на входе автотрансформатора. Очевидно, схемы на рис. 4, $a$ и 6 , а также рис. 7 $а$ эквивалентны по мощностям.

\section{Дубликаты схем замещения автотрансформатора}

В схеме на рис. 7, $a$ величина $L_{\text {кз }}^{b}-$ индуктивность К3, приведенная к обмотке с числом витков $w_{b}$. Используя подстановку $L_{\text {к3 }}^{b}=n_{a}^{b 2} L_{\text {к3 }}^{a}$, получаем эквивалентную схему (рис. 7,6 ), в которой $L_{\mathrm{K} 3}^{a}-$ индуктивность К3, приведенная к обмотке с числом витков $w_{a}$. Все напряжения и токи остаются прежними. Очевидно, таким же образом можно получить дубликаты схем (рис. 4 и рис. 5), что весьма важно при сопоставлении схемных моделей автотрансформаторов, приводимых различными авторами. Для этой же цели полезно на схемах штрихованные величины

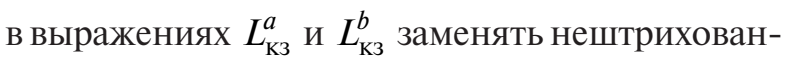
ными. Согласно табл. 1 можем записать

$$
\begin{aligned}
& L_{\mathrm{K} 3}^{a}=L_{a}+L_{\delta}^{a}+n_{b}^{a} L_{b} ; \\
& L_{\mathrm{K} 3}^{b}=n_{a}^{b 2} L_{a}+L_{\delta}^{b}+L_{b} .
\end{aligned}
$$

\section{Сравнение свернутых схем замещения со схемами в учебниках}

В схеме на рис. 7, б продольную индуктивность можно представить следующим образом:

$$
n_{b}^{2} L_{\text {к } 3}^{a}=n_{b}^{2} L_{a}+n_{b}^{2} L_{\delta}^{a}+n_{b}^{2}\left(n_{b}^{a 2} L_{b}\right) .
$$

Разобьем, как это принято в традиционной теории, индуктивность $L_{\delta}^{b}$ между обмотками, отнеся некоторую ее часть $\left(\lambda L_{\delta}^{b}\right)$ к обмотке $w_{a}$, а другую часть $(1-\lambda) L_{\delta}^{b}-$ к обмотке $w_{b}$, где $\lambda-$ любое число, не равное нулю. Тогда можно записать

$$
\begin{gathered}
n_{b}^{2} L_{\text {К } 3}^{a}=n_{b}^{2} L_{a}+\lambda n_{b}^{2} L_{\delta}^{a}+(1-\lambda) n_{b}^{2} L_{\delta}^{a}+n_{b}^{2} n_{b}^{a 2} L_{b}= \\
=n_{b}^{2}\left(L_{a}+\lambda L_{\delta}^{a}\right)+(1-\lambda) n_{b}^{2} n_{b}^{a 2} L_{\delta}^{b}+n_{b}^{2} n_{b}^{a 2} L_{b} .
\end{gathered}
$$

Обозначив

$$
\begin{gathered}
L_{\alpha}=\left(L_{a}+\lambda L_{\delta}^{a}\right), \\
L_{s}=(1-\lambda) L_{\delta}^{b}+L_{b}
\end{gathered}
$$

и учтя, что

$$
n_{b} n_{b}^{a}=n_{a}, n_{b}=1-n_{a},
$$

продольную индуктивность в схеме на рис. $7, \sigma$ перепишем в виде

$$
n_{b}^{2} L_{\mathrm{K} 3}^{a}=\left(1-n_{a}\right)^{2} L_{\alpha}+n_{a}^{2} L_{s} .
$$

Этот результат показывает, что схема на рис. 7, 6 совпадает со схемой, представленной в [7, стр.79, рис. $3-4, \kappa]$, если в ней переобозначить $k^{\prime}=n_{a}$. Тот факт, что этот вывод не зависит от выбора числа $\lambda$, подчеркивает бессмысленность разбиения индуктивности $L_{\delta}^{b}$ между обмотками, 

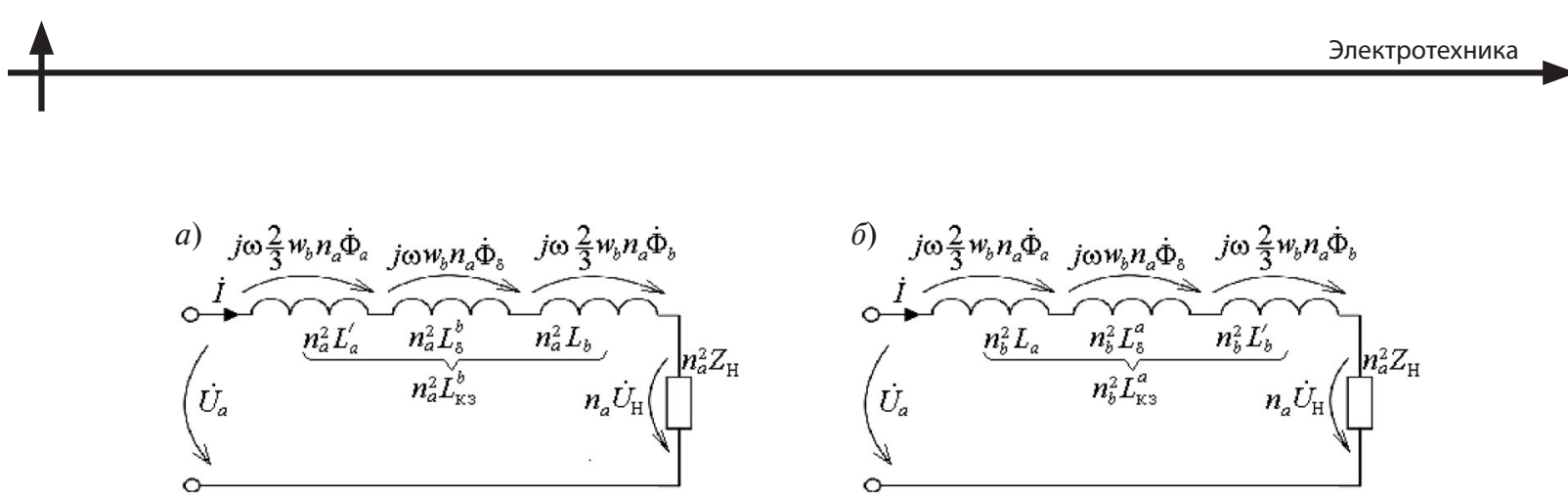

Рис. 7. Упрощенная схема замещения повышающего автотрансформатора относительно входных величин тока $\dot{I}, \dot{U}_{a}$ с индуктивностью КЗ $n_{a}^{2} L_{\text {к3 }}^{b}(a)$ и ее дубликат с индуктивностью КЗ $n_{b}^{2} L_{\text {кз }}^{a}$ (б)

а вместе с этим и слабую обоснованность традиционной теории автотрансформаторов.

Схема на рис. 7, б совпадает также со схемой замещения для повышающего автотрансформатора, приведенной в учебнике [4, с. 224], в котором продольное сопротивление представлено формулой под номером (11-23):

$$
\begin{aligned}
Z_{\ni}= & Z_{k} k^{2}=\left(Z_{\Pi}+Z / k_{A}^{2}\right) k^{2}=2 \\
& =k^{2} Z_{\Pi}+\left(k / k_{A}\right)^{2} Z .
\end{aligned}
$$

Выполнив переобозначения

$$
\begin{gathered}
k_{A}=n_{b}^{a}, \quad k=n_{a}, \quad Z_{\text {п }}=j \omega L_{s}, \quad Z=j \omega L_{\alpha}, \\
Z_{k}=j \omega L_{\text {к3 }}^{b},
\end{gathered}
$$

ее можно переписать в виде

$$
Z_{\ni}=j \omega n_{a}^{2} L_{\text {К }}^{b}=j \omega\left(n_{a}^{2} L_{s}+\left(n_{a} / n_{b}^{a}\right)^{2} L_{\alpha}\right),
$$

или

или

$$
Z_{\text {э }}=j \omega n_{a}^{2} n_{a}^{b^{2}} L_{\mathrm{K} 3}^{a}=j \omega\left(n_{a}^{2} L_{s}+\left(n_{a} n_{a}^{b}\right)^{2} L_{\alpha}\right),
$$

$$
Z_{\ni}=j \omega\left(n_{a} n_{a}^{b}\right)^{2} L_{\mathrm{K} 3}^{a}=j \omega\left(n_{a}^{2} L_{s}+n_{b}^{2} L_{\alpha}\right),
$$

или

$$
Z_{\ni}=j \omega n_{b}^{2} L_{\mathrm{K} 3}^{a}=j \omega\left(n_{a}^{2} L_{s}+\left(1-n_{A}\right)^{2} L_{\alpha}\right),
$$

что отличается от уравнения (33) лишь множителем $j \omega$ и подтверждает тождественность схем замещения авторов работ [7] и [4]. Данный пример подтверждает сложность и разнобой в обозначениях, применяемых различными авторами учебников. Совпадение схем замещения на рис. 7, б со схемами в учебниках свидетельствует о достоверности последних только для идеализированного автотрансформатора. Вместе с тем схемы на рис. 7 информативнее учебных, поскольку позволяют одновременно с электри- ческим величинами определять потоки в окне автотрансформатора.

\section{Режим холостого хода идеализированного автотрансформатора}

В режиме $\mathrm{XX}$ сопротивление нагрузки $Z_{\mathrm{H}}=\infty$, и согласно любой схеме замешения

$$
2 \quad \dot{I}_{a}^{\mathrm{xx}}=\dot{I}_{b}^{\mathrm{xx}}=\dot{I}^{\mathrm{xx}}=0 .
$$

Поэтому и потоки в окне будут нулевыми:

$$
\dot{\Phi}_{a}^{\mathrm{xx}}=\dot{\Phi}_{\delta}^{\mathrm{xx}}=\dot{\Phi}_{b}^{\mathrm{xx}}=0 ; \dot{\Phi}_{\mathrm{OKH}}^{\mathrm{xx}}=0 .
$$

С учетом этого по схемам замещения на рис. 3, б, в убеждаемся, что все потоки XX в магнитопроводе одинаковы:

$$
\dot{\Phi}_{\mathrm{xx}}=\dot{\Phi}_{\mathrm{cT}}^{\mathrm{xx}}=\dot{\Phi}_{\mathrm{q}}^{a^{\mathrm{xx}}}=\dot{\Phi}_{\mathrm{g}}^{b^{\mathrm{xx}}}=\dot{\Phi}_{\text {боК }}^{\mathrm{xx}}=\frac{\dot{U}_{a}}{j \omega w_{a}} .
$$

Для напряжений по этим схемам имеем

$$
\begin{gathered}
\dot{U}_{b}^{\mathrm{xx}}=n_{a}^{b} \dot{U}_{a}=\frac{\dot{U}_{a}}{n_{b}^{a}}=\frac{w_{b}}{w_{a}} \dot{U}_{a} ; \\
\dot{U}_{\mathrm{H}}^{\mathrm{xx}}=\dot{U}_{a}+\dot{U}_{b}^{\mathrm{xx}}=\frac{\dot{U}_{a}}{n_{a}}=\frac{\dot{U}_{a}}{1-n_{b}} .
\end{gathered}
$$

Можно также записать

$$
\dot{U}_{\mathrm{H}}^{\mathrm{xx}}=\frac{\dot{U}_{b}^{\mathrm{xx}}}{n_{a}^{b} n_{a}}=\frac{\dot{U}_{b}^{\mathrm{xx}}}{n_{b}} .
$$

\section{Режим короткого замыкания}

Анализ установившегося режима К3 автотрансформатора при номинальном напряжении $\dot{U}_{a}$ на входе важен как для понимания физических основ его работы при перегрузках, так и для выявления сверх- и антипотоков в магнитопроводе при $Z_{\mathrm{H}}=0$. В этом случае для токов К3 согласно (23), (18) и (25) можно написать 


$$
\begin{gathered}
\dot{I}_{a}^{\mathrm{K} 3}=\frac{1}{n_{b}} \frac{\dot{U}_{a}}{j \omega L_{\mathrm{K} 3}^{a}} ; \quad \dot{I}_{b}^{\mathrm{K} 3}=n_{b}^{a} \dot{I}_{a}^{\mathrm{K} 3}=\frac{1}{n_{a}} \frac{\dot{U}_{a}}{j \omega L_{\mathrm{K} 3}^{b}} ; \\
\dot{I}^{\mathrm{K} 3}=\frac{\dot{U}_{a}}{n_{a}^{2} j \omega L_{\mathrm{K} 3}^{b}} .
\end{gathered}
$$

Непосредственно из схем рис. 3, 6, в следует

$$
j \omega w_{a} \dot{\Phi}_{a}^{\mathrm{K} 3}=j \omega \frac{3}{2} L_{a} \dot{I}_{a}^{\mathrm{K} 3},
$$

откуда для потока в толще обмотки $w_{a}$ получаем

$$
\begin{gathered}
\dot{\Phi}_{a}^{\mathrm{K} 3}=\frac{3}{2} L_{a} \frac{\dot{I}_{a}^{\mathrm{K} 3}}{w_{a}}=\frac{3}{2} L_{a} \frac{\dot{U}_{a}}{n_{b} j \omega L_{\mathrm{K} 3}^{a} w_{a}}= \\
=3 \frac{1}{n_{b}} \frac{L_{a}}{2 L_{\mathrm{K} 3}^{a}} \frac{\dot{U}_{a}}{j \omega w_{a}}=3 \frac{1}{n_{b}} \frac{L_{a}}{2 L_{\mathrm{K} 3}^{a}} \dot{\Phi}_{\mathrm{xx}},
\end{gathered}
$$

или в относительных единицах

$$
\dot{\Phi}_{a}^{\mathrm{K} 3}=\frac{\dot{\Phi}_{a}^{\mathrm{K} 3}}{\dot{\Phi}_{\mathrm{xx}}}=\frac{3}{n_{b}} \frac{L_{a}}{2 L_{\mathrm{K} 3}^{a}} .
$$

Найдем аналогичное соотношение для потока в стержне $\dot{\Phi}_{\text {ст }}^{\text {к3 }}$. Пользуясь вторым законом Кирхгофа по схемам на рис. $3,6,6$, имеем

$$
-j \omega \frac{1}{2} L_{a} \dot{I}_{a}^{\mathrm{K} 3}+j \omega w_{a} \dot{\Phi}_{\mathrm{cT}}^{\mathrm{K} 3}=\dot{U}_{a},
$$

или

$$
\begin{gathered}
j \omega w_{a} \dot{\Phi}_{\mathrm{cT}}^{\mathrm{K} 3}=\dot{U}_{a}+j \omega \frac{1}{2} L_{a} \dot{I}_{a}^{\mathrm{K} 3}= \\
=\dot{U}_{a}+j \omega \frac{1}{2} L_{a} \frac{1}{n_{b}} \frac{\dot{U}_{a}}{j \omega L_{\mathrm{K} 3}^{a}} .
\end{gathered}
$$

После сокращений и деления обеих частей равенства на $j \omega w_{a}$ получаем

$$
\dot{\Phi}_{\mathrm{cT}}^{\mathrm{K} 3}=\left(1+\frac{1}{n_{b}} \frac{L_{a}}{2 L_{\mathrm{K} 3}^{a}}\right) \frac{\dot{U}_{a}}{j \omega w_{a}}=\left(1+\frac{1}{n_{b}} \frac{L_{a}}{2 L_{\mathrm{K} 3}^{a}}\right) \dot{\Phi}_{\mathrm{xx}} .
$$

Таким образом,

$$
\dot{\Phi}_{\mathrm{cT}}^{\mathrm{K} 3}=\frac{\dot{\Phi}_{\mathrm{cT}}^{\mathrm{K} 3}}{\dot{\Phi}_{\mathrm{xx}}}=\left(1+\frac{1}{n_{b}} \frac{L_{a}}{2 L_{\mathrm{K} 3}^{a}}\right) .
$$

Чтобы найти поток К3 $\dot{\Phi}_{я}^{b^{\text {К3 }}}$, воспользуемся его общим выражением (19), положив сопротивление нагрузки равным нулю:

$\dot{\Phi}_{\text {я }}^{b^{\mathrm{K} 3}}=\left(\frac{j \omega L_{b}}{n_{a} j \omega L_{\mathrm{K} 3}^{b}}-1\right) \frac{\dot{U}_{a}}{j \omega w_{b}}=\left(\frac{L_{b}}{n_{a} L_{\mathrm{K} 3}^{b}}-1\right) \frac{\dot{U}_{a}}{j \omega w_{a} n_{a}^{b}}=$

$$
=\left(\frac{L_{b}}{n_{a} L_{\mathrm{K} 3}^{b}}-1\right) \frac{1}{n_{a}^{b}} \dot{\Phi}_{\mathrm{xx}},
$$

откуда

$$
\underline{\dot{\Phi}}_{\Upsilon}^{b^{\mathrm{K} 3}}=\frac{\dot{\Phi}_{\varsigma}^{b^{\mathrm{K}} 3}}{\dot{\Phi}_{\mathrm{xX}}}=\frac{1}{n_{a}^{b}}\left(\frac{1}{n_{a}} \frac{L_{b}}{L_{\mathrm{K} 3}^{b}}-1\right)=\left(\frac{1}{n_{b}} \frac{L_{b}^{\prime}}{L_{\mathrm{K} 3}^{a}}-n_{b}^{a}\right) .
$$

Аналогичным образом нетрудно получить и другие потоки автотрансформатора при К3 (табл. 3).

Как и следовало ожидать, при К3 имеет место следующее равенство для действующих значений потоков:

$$
\Phi_{\mathrm{OKH}}^{\mathrm{K} 3}=\Phi_{\mathrm{CT}}^{\mathrm{K} 3}+\Phi_{\text {боК }}^{\mathrm{K} 3} .
$$

Для расчета потоков К3 по формулам табл. 3 необходим предварительный расчет отношения индуктивностей к индуктивности К3, что нетрудно сделать, воспользовавшись соотношениями табл. 1 и формулами (1), (3)-(5). Например,

$$
\begin{gathered}
\frac{L_{a}}{L_{\text {к3 }}^{a}}=\frac{L_{a}^{\prime}}{L_{\text {к3 }}^{b}}=\frac{w_{a}^{\mathrm{M}} / 3 R_{a}}{\frac{w_{a}^{2}}{3 R_{a}^{\mathrm{M}}}+\frac{w_{a}^{2}}{R_{\delta}^{\mathrm{M}}}+\frac{w_{a}^{2}}{3 R_{b}^{\mathrm{M}}}}= \\
=\frac{s_{a}}{s_{a}+3 s_{\delta}+s_{b}}=\frac{a D_{a}}{a D_{a}+3 a D_{\delta}+b D_{b}} .
\end{gathered}
$$

Если принять $D_{a} \approx D_{\delta} \approx D_{b}$, то после сокращений получаем приближенное выражение

$$
\frac{L_{a}}{L_{\mathrm{K} 3}^{a}}=\frac{L_{a}^{\prime}}{L_{\mathrm{K} 3}^{b}}=\frac{a}{a+3 a+b} .
$$

Аналогично получаются приближенные выражения для отношений остальных индуктивностей (табл. 4).

3 а меч а н и е 1 . В случае бесконечно тонких обмоток ( $a=0, b=0)$ имеем

$$
L_{a} / L_{\mathrm{K} 3}^{a}=0, L_{\delta} / L_{\mathrm{K} 3}^{a}=1, L_{b} / L_{\mathrm{K} 3}^{a}=0,
$$

и из табл. 3 следует, что в этом случае магнитные потоки при К3 будут такими:

$$
\begin{gathered}
\dot{\Phi}_{\mathrm{cT}}^{\mathrm{K} 3}=\dot{\Phi}_{Я}^{a^{\mathrm{K} 3}}=\dot{\Phi}_{\mathrm{xX}} ; \\
\dot{\Phi}_{\text {боК }}^{\mathrm{K} 3}=\dot{\Phi}_{\text {Я }}^{b^{\mathrm{K} 3}}=-n_{b}^{a} \dot{\Phi}_{\mathrm{xX}} ; \\
\dot{\Phi}_{a}^{\mathrm{K} 3}=\dot{\Phi}_{b}^{\mathrm{K} 3}=0 ;
\end{gathered}
$$


Таблица 3

Магнитные потоки в идеализированном автотрансформаторе в режиме К3

\begin{tabular}{|c|c|c|}
\hline $\begin{array}{c}\text { Область (участок) } \\
\text { автотрансформатора }\end{array}$ & $\begin{array}{c}\text { Поток } k \text {-го участка, } \\
\text { отнесенный к потоку XX } \\
\left(\underline{\Phi}_{k}^{\text {К3 }}=\frac{\dot{\Phi}_{k}^{\text {К3 }}}{\dot{\Phi}_{\mathrm{xx}}}\right)\end{array}$ & Примечание \\
\hline Стержень & $\underline{\Phi}_{\mathrm{cT}}^{\mathrm{K} 3}=1+\frac{1}{n_{b}} \frac{L_{a}}{2 L_{\mathrm{K} 3}^{a}}$ & $\begin{array}{l}\dot{\Phi}_{\mathrm{cT}}^{\mathrm{K}}-\text { сверхпоток, } \\
\text { так КаК } \Phi_{\mathrm{cT}}^{\mathrm{K} 3}>\Phi_{\mathrm{xx}}\end{array}$ \\
\hline Левый участок стыкового ярма & $\underline{\dot{\Phi}}_{\Upsilon}^{a^{\mathrm{K} 3}}=1-\frac{1}{n_{b}} \frac{L_{a}}{L_{\mathrm{K} 3}^{a}}$ & - \\
\hline Правый участок стыкового ярма & $\dot{\Phi}_{\Upsilon}^{b^{\mathrm{K} 3}}=\frac{1}{n_{b}} \frac{L_{b}^{\prime}}{L_{\mathrm{K} 3}^{a}}-n_{b}^{a}$ & - \\
\hline Боковое ярмо & $\underline{\dot{\Phi}}_{\text {бок }}^{\text {Кз }}=-\left(n_{b}^{a}+\frac{1}{n_{b}} \frac{L_{b}^{\prime}}{2 L_{\mathrm{K} 3}^{a}}\right)$ & $\begin{array}{c}\dot{\Phi}_{\text {бок }}^{\text {к3 }}-\text { антипоток, } \\
\text { (так как направлен встречно } \\
\left.\text { потоку в стержне } \dot{\Phi}_{\mathrm{cт}}^{\text {к3 }}\right)\end{array}$ \\
\hline Внутренняя обмотка & $\dot{\Phi}_{a}^{\mathrm{K} 3}=\frac{\dot{\Phi}_{a}^{\mathrm{K} 3}}{\dot{\Phi}_{\mathrm{xx}}}=\frac{3}{n_{b}} \frac{L_{a}}{2 L_{\mathrm{K} 3}^{a}}$ & - \\
\hline Коридор между обмотками & $\dot{\Phi}_{\delta}^{\mathrm{K} 3}=\frac{\dot{\Phi}_{\delta}^{\mathrm{K} 3}}{\dot{\Phi}_{\mathrm{xx}}}=\frac{L_{\delta}^{a}}{n_{b} L_{\mathrm{K} 3}^{a}}$ & - \\
\hline Внешняя обмотка & $\underline{\Phi}_{b}^{\mathrm{K} 3}=\frac{\dot{\Phi}_{a}^{\mathrm{K} 3}}{\dot{\Phi}_{\mathrm{xx}}}=\frac{3}{n_{b}} \frac{L_{b}^{\prime}}{2 L_{\mathrm{K} 3}^{a}}$ & - \\
\hline Окно & $\underline{\Phi}_{\mathrm{OKH}}^{\mathrm{K} 3}=\frac{\dot{\Phi}_{\mathrm{OKH}}^{\mathrm{K} 3}}{\dot{\Phi}_{\mathrm{XX}}}=\frac{1}{n_{b}}\left(1+\frac{L_{a}+L_{b}^{\prime}}{2 L_{\mathrm{K} 3}^{a}}\right)$ & $\begin{array}{c}\text { Поток в окне больше } \\
\text { сверхпотока в стержне: } \\
\Phi_{\mathrm{OKH}}^{\mathrm{K3}}>\Phi_{\mathrm{cт}}^{\mathrm{K3}}>\Phi_{\mathrm{xx}}\end{array}$ \\
\hline
\end{tabular}

Таблица 4

Отношения индуктивностей при $h_{\text {об }}=h_{\text {окн }}$

\begin{tabular}{|c|c|c|}
\hline Относителльная величина & Формула & Приближенная формула \\
\hline$\frac{L_{a}}{L_{\mathrm{K} 3}^{a}}=\frac{L_{a}^{\prime}}{L_{\mathrm{K} 3}^{b}}$ & $\frac{a D_{a}}{a D_{a}+3 \delta D_{\delta}+b D_{b}}$ & $\frac{a}{a+3 \delta+b}$ \\
\hline$\frac{L_{\delta}^{a}}{L_{\mathrm{K} 3}^{a}}=\frac{L_{\delta}^{b}}{L_{\mathrm{K} 3}^{b}}$ & $\frac{3 \delta D_{\delta}}{a D_{a}+3 \delta D_{\delta}+b D_{b}}$ & $\frac{3 \delta}{a+3 \delta+b}$ \\
\hline$\frac{L_{b}^{\prime}}{L_{\mathrm{K} 3}^{a}}=\frac{L_{b}}{L_{\mathrm{K} 3}^{b}}$ & $\frac{b D_{b}}{a D_{a}+3 \delta D_{\delta}+b D_{b}}$ & $\frac{b}{a+3 \delta+b}$ \\
\hline
\end{tabular}




$$
\dot{\Phi}_{\delta}^{\mathrm{K} 3}=\dot{\Phi}_{\mathrm{OKH}}^{\mathrm{K} 3}=\frac{1}{n_{b}} \dot{\Phi}_{\mathrm{xX}}=\left(1+n_{b}^{a}\right) \dot{\Phi}_{\mathrm{xx}} .
$$

Из этих формул следует, что даже в случае тонких обмоток в боковом ярме при К 3 внешней обмотки возникает внушительный антипоток, существенно (при больших $\left.n_{b}^{a}=\left(w_{a}+w_{b}\right) / w_{b}\right)$ превышающий поток $\mathrm{XX}\left(\dot{\Phi}_{\mathrm{xx}}\right)$ со стороны обмотки $w_{a}$. Это объясняется тем, что последовательная обмотка $\left(w_{b}\right)$ при К3 , как и первичная $\left(w_{a}\right)$ обмотка, оказывается включенной непосредственно на входное напряжение $\dot{U}_{a}$, что приводит к появлению в боковом ярме потока $\dot{\Phi}_{\text {бок }}^{\mathrm{K} 3}=\dot{\Phi}_{\mathrm{xx}}^{b}$, где $\dot{\Phi}_{\mathrm{xx}}^{b}-$ поток XX со стороны обмотки $w_{b}$, равный

$$
\dot{\Phi}_{\mathrm{xx}}^{b}=\frac{\dot{U}_{a}}{j \omega w_{b}}=\frac{\dot{U}_{a}}{j \omega w_{a}} \frac{w_{a}}{w_{b}}=\dot{\Phi}_{\mathrm{xx}} n_{b}^{a} .
$$

В соответствии с (44) оба потока (со стороны стержня и бокового ярма) устремляются в межобмоточное пространство, создавая тем самым сверхпоток в окне между обмотками $\dot{\Phi}_{\delta}^{\mathrm{K} 3}=\left(1+n_{b}^{a}\right) \dot{\Phi}_{\mathrm{xx}}$. Учет конечной толщины обмоток еще более усиливает эти эффекты.

Пример. Рассмотрим идеализированный повышающий автотрансформатор AT1, построенный на базе трансформатора Tp1 [18], со следующими данными: $w_{a}=535, w_{b}=161, a=10$ мм, $b=3 \mathrm{мM}, \delta \approx 0, h_{\text {об }}=h_{\text {окн }}$. Из этих данных следует

$$
\begin{gathered}
n_{b}^{a}=\frac{w_{a}}{w_{b}}=\frac{535}{161}=3,32 ; \\
n_{b}=\frac{w_{b}}{w_{a}+w_{b}}=\frac{161}{535+161}=0,231 ; \\
\frac{1}{n_{b}}=\frac{1}{0,23}=4,32 .
\end{gathered}
$$

В режиме $\mathrm{XX}$

$$
\begin{gathered}
\dot{U}_{b}^{\mathrm{xx}}=\frac{\dot{U}_{a}}{n_{b}^{a}}=\frac{\dot{U}_{a}}{3,32}=0,3 \dot{U}_{a} ; \\
\dot{U}_{\mathrm{H}}^{\mathrm{xx}}=\frac{\dot{U}_{a}}{1-n_{b}}=\frac{\dot{U}_{a}}{1-0,231}=1,3 \dot{U}_{a} .
\end{gathered}
$$

Для расчета режима К3 определяем следующие величины:

$$
\frac{L_{a}}{L_{\text {К } 3}^{a}} \approx \frac{a}{(a+3 \delta+b)}=\frac{10}{10+3 \cdot 0+3}=0,77 ;
$$

$$
\begin{gathered}
\frac{L_{b}^{\prime}}{L_{\mathrm{K} 3}^{a}} \approx \frac{b}{a+3 \delta+b}=\frac{3}{10+3 \cdot 0+3}=0,23 ; \\
\frac{L_{\delta}}{L_{\mathrm{K} 3}^{a}} \approx \frac{3 \delta}{a+3 \delta+b}=0 .
\end{gathered}
$$

Расчеты потоков представлены во второй колонке табл. 5 для обмоток заданной толщины. Как видно, во всех рассматриваемых участках магнитопровода и окна, за исключением промежутка между обмотками (в силу его отсутствия, т. к. $\delta=0$ ), при К3 имеет место значительное превышение магнитного потока XX. Антипотоки возникли и в участках стыковых ярем с заходом во внутреннюю обмотку. Превышение этих потоков над потоком XX дает основание называть их антисверхпотоками.

Во второй колонке табл. 5 приведены экспериментальные данные, найденные с помощью измерительных витков, намотанных на стержень и боковое ярмо АТ1. Поток в окне получен суммированием значений потоков в стержне и боковом ярме. Погрешность расчета и эксперимента не превышает $17 \%$.

В третьей колонке табл. 5 представлены результаты расчета потоков для случая тонких обмоток. Их сравнение с данными первой колонки показывает, что в общем случае значения потоков при КЗ весьма существенно зависят от геометрии магнитопровода и обмоток.

\section{4Т-образная схемная модель реального автотрансформатора}

В отличие от идеализированного, в реальном автотрансформаторе имеют место потери в меди обмоток и стали магнитопровода с конечной магнитной проницаемостью. Их можно учесть приближенно путем наращивания полученных выше схем замещения идеализированного автотрансформатора (см. рис. 3) активными сопротивлениями $R_{a}, R_{b}$ обмоток, а также поперечными ветвями для учета активных и реактивных потерь в стали, включая участки между баком и частями магнитопровода, как показано пунктиром на схеме (рис. $8, a$ ), образованной из модели на рис. 3 , б. В ней нелинейные индуктивности и включенные параллельно с ними активные проводимости соответствуют следующим элементам:

$L_{\text {я }}^{a}, g_{\text {я }}^{a}-$ части стыкового ярма, примыкающей к стержню; 
Магнитные потоки в идеализированном автотрансформаторе Ат1 в режиме КЗ

\begin{tabular}{|c|c|c|c|}
\hline $\begin{array}{c}\text { Область (участок) } \\
\text { автотрансформатора АТ1 }\end{array}$ & $\begin{array}{c}\text { Поток } k \text {-го участка, отнесенный } \\
\text { к потоку ХХ, } \\
\left(\underline{\dot{\Phi}}_{k}^{\text {Кз }}=\frac{\dot{\Phi}_{k}^{\text {к3 }}}{\dot{\Phi}_{\mathrm{xx}}}\right), \\
\text { в случае обмоток конечной } \\
\text { толщины }(a=10 \text { мм, } b=3 \text { мм, } \delta=0)\end{array}$ & $\begin{array}{c}\text { Экспери- } \\
\text { мент }\end{array}$ & $\begin{array}{c}\text { Поток } k \text {-го участка, } \\
\text { отнесенный к потоку ХХ, } \\
\left(\underline{\dot{\Phi}}_{k}^{\text {К3 }}=\frac{\dot{\Phi}_{k}^{\text {К3 }}}{\dot{\Phi}_{\text {хх }}}\right), \\
\text { в случае тонких обмоток } \\
(a=b=0, \delta \neq 0)\end{array}$ \\
\hline Стержень & $\dot{\Phi}_{\mathrm{cT}}^{\mathrm{K} 3}=1+4,32 \frac{0,77}{2}=2,66$ & 2,77 & 1 \\
\hline $\begin{array}{l}\text { Левый участок стыкового } \\
\text { ярма }\end{array}$ & $\dot{\Phi}_{\mathrm{\Upsilon}}^{a^{\mathrm{K} 3}}=1-4,32 \cdot 0,77=-2,32$ & - & 1 \\
\hline $\begin{array}{l}\text { Правый участок стыкового } \\
\text { ярма }\end{array}$ & $\dot{\Phi}_{9}^{b^{\mathrm{K} 3}}=4,32 \cdot 0,23-3,32=-2,32$ & - & $-3,32$ \\
\hline Боковое ярмо & $\dot{\Phi}_{\text {боK }}^{\mathrm{K} 3}=-\left(3,32+4,32 \frac{0,23}{2}\right)=-3,82$ & $-3,03$ & $-3,32$ \\
\hline Внутренняя обмотка & $\dot{\Phi}_{a}^{\mathrm{K} 3}=3 \cdot 4,32 \cdot \frac{0,77}{2}=4,99$ & - & 0 \\
\hline Коридор между обмотками & $\dot{\Phi}_{\delta}^{\mathrm{K} 3}=0$ & - & 4,32 \\
\hline Внешняя обмотка & $\dot{\Phi}_{b}^{\mathrm{K} 3}=\frac{3}{0,231} \frac{0,23}{2}=1,49$ & - & 0 \\
\hline Окно & $\dot{\Phi}_{\mathrm{OKH}}^{\mathrm{K} 3}=4,32\left(1+\frac{0,77+0,23}{2}\right)=6,48$ & 5,8 & 4,32 \\
\hline
\end{tabular}

a)
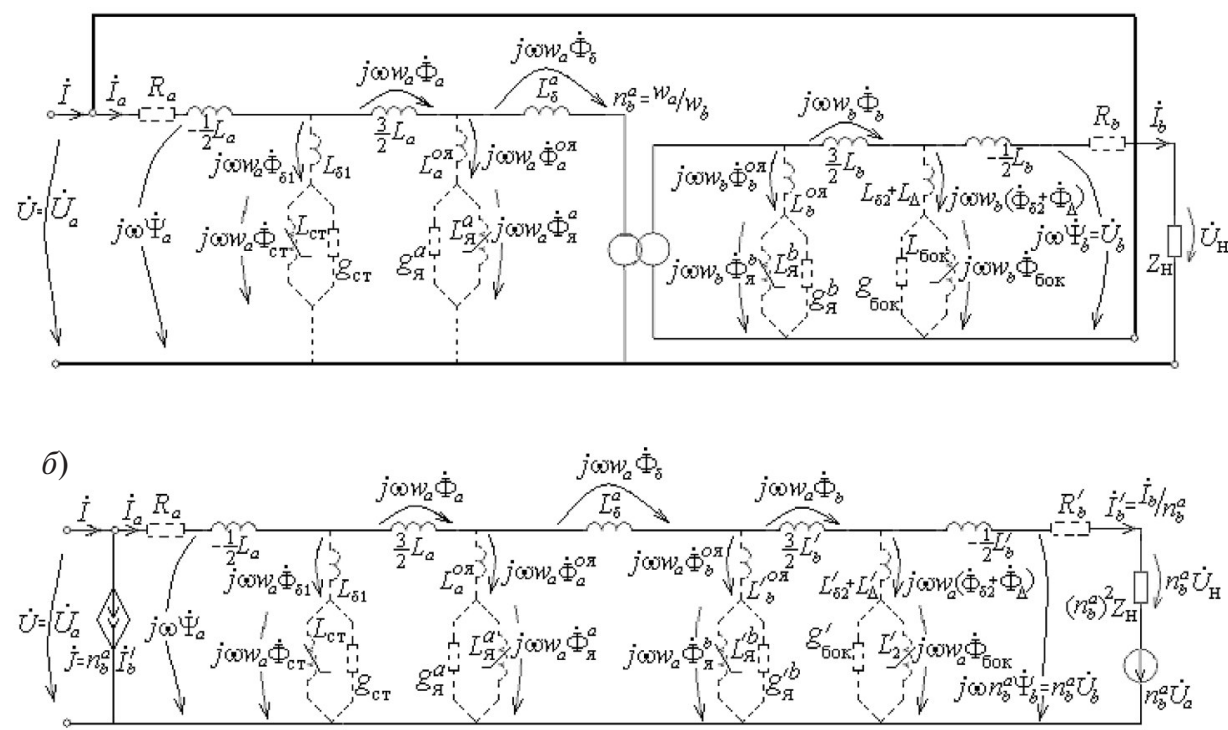

Рис. 8. Эвристическая схема замещения реального автотрансформатора ( $a$ ) и его модель после приведения вторичной обмотки $w_{b}$ к первичной $w_{a}($ б) 
$L_{\text {я }}^{b}, g_{\text {я }}^{b}\left(L_{я}^{\prime b}, g_{я}^{\prime b}\right)-$ части стыкового ярма, примыкающей к боковому ярму;

$L_{\text {ст }}, g_{\text {ст }}-$ стержню;

$L_{\text {бок }}, g_{\text {бок }}\left(L_{\text {бок }}^{\prime}, g_{\text {бок }}^{\prime}\right)-$ боковому ярму.

Последовательно присоединенные к ним линейные индуктивности

$$
\begin{gathered}
L_{\delta 1}=\frac{\mu_{0} s_{\delta 1}}{h} w_{a}^{2}, \quad L_{a}^{o Я}=\frac{\mu_{0} s_{a}^{o Я}}{l_{a}^{o Я}} w_{a}^{2}, \\
L_{b}^{o Я}=\frac{\mu_{0} s_{b}^{o Я ~}}{l_{b}^{o Я}} w_{b}^{2}, \quad L_{\delta 2}=\frac{\mu_{0} s_{\delta 2}}{h} w_{b}^{2}, \quad L_{\Delta}=\frac{\mu_{0} s_{\Delta}}{h} w_{b}^{2}
\end{gathered}
$$

введены для учета магнитных потоков $\dot{\Phi}_{\delta 1}, \dot{\Phi}_{a}^{o я}$, $\dot{\Phi}_{b}^{o Я}, \dot{\Phi}_{\delta 2}, \dot{\Phi}_{\Delta}$ (см. рис. 2), возникающих вследствие конечной магнитной проницаемости стали или ее насыщения (смысл значений площадей $s_{a}^{o я}$ ,$s_{b}^{o Я}$ и длин $l_{a}^{o Я}, l_{a}^{o Я ~ о ч е в и д е н ~ и з ~ р а с с м о т р е н и я ~}$ потоков $\left.\dot{\Phi}_{a}^{o Я}, \dot{\Phi}_{b}^{o Я}\right)$. Штрихами отмечены величины в схеме рис. $8, \sigma$, приведенные к обмотке $w_{a}$.

П р и м е ч а н и е. Добавление поперечных ветвей основано на известном из теории магнитных цепей положении, согласно которому ферромагнитному сердечнику катушки (с числом витков $w$ ), в котором протекает поток $\dot{\Phi}_{k}$, на схеме замещения катушки соответствует активная проводимость $g_{k}$ и нелинейная индуктивная проводимость $b_{k}$, удовлетворяющие соотношениям

$$
g_{k}=\frac{P_{F e_{k}}}{\left(\omega w \Phi_{k}\right)^{2}} ; \quad b_{k}=\frac{1}{\omega L_{k}\left(\Phi_{k}\right)},
$$

где $P_{F_{k}}$ - активные потери в сердечнике (здесь в $k$-м участке магнитопровода) и $L_{k}\left(\Phi_{k}\right)=\frac{w^{2}}{R_{k}^{\mathrm{M}}\left(\Phi_{k}\right)}-$ нелинейная индуктивность; $R_{k}^{\mathrm{M}}\left(\Phi_{k}\right)-$ нелинейное магнитное сопротивление стали на $k$-м участке магнитопровода.

Поперечная ветвь, состоящая из элементов $L_{1}, g_{1}, L_{\delta 1}$, нарушает пропорцию между напряже- ниями на продольных индуктивностях $\left(-L_{a} / 2\right)$ и $\left(3 L_{a} / 2\right)$, имевшую место в исходных схемах (см. рис. 3). Поэтому на рис. 8, $a$ явное представление напряжения на индуктивности $\left(-L_{a} / 2\right)$ через поток $\dot{\Phi}_{a} / 3$ устранено. По аналогичной причине устранено представление через поток $\dot{\Phi}_{b} / 3$ напряжения на индуктивности $\left(-L_{b} / 2\right)$ и на индуктивности $-L_{b}^{\prime} / 2$. Несмотря на полуэвристический характер полученной 4Т-образной схемы (рис. 8, $a$ ), уравнения, составленные по второму закону Кирхгофа для напряжений, показанных на схеме стрелками, в точности соответствуют соотношениям для магнитных потоков (см. рис. 2).

Приведенная 4Т-образная схема замещения реального автотрансформатора (рис. 8, б), построена на базе приведенной схемы для идеализированного автотрансформатора (см. рис. 10 в Приложении).

Эта же схема с выделенным общим током намагничивания $\dot{I}_{0}$ показана на рис. 9. В принципе ее среднюю часть можно преобразовать в эквивалентную звезду или треугольник. Из нее могут быть выведены Т-, П- и Г-образные схемные модели.

\section{Заключение}

Изложенная работа имеет практическую направленность в свете весьма печальной статистики о росте повреждений и выходе из строя автотрансформаторов в аварийных ситуациях [21]. Из нее следует, что принимаемые до сих пор попытки повысить электродинамическую стойкость автотрансформаторов путем устранения дефектов производственного характера, т. е. улучшением механических характеристик обмоток или изоляции и применением новых, нетрадиционных материалов, недостаточны. Необходимы шаги по усовершенствованию теории автотрансформаторов в силу отсутствия в тра-

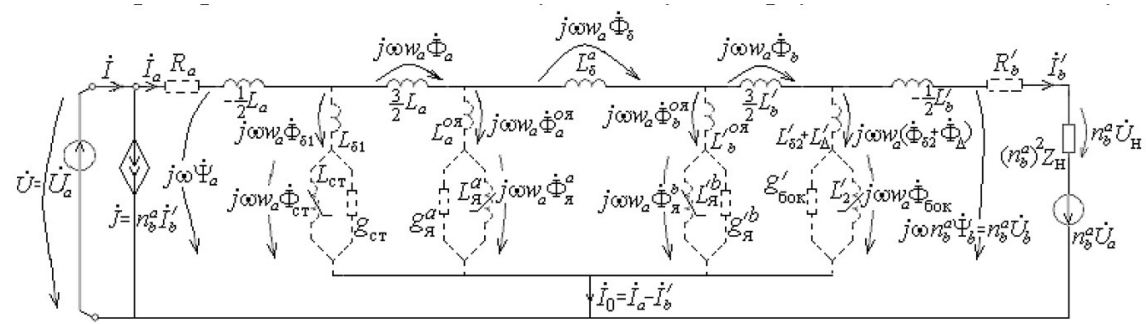

Рис. 9. Приведенная 4Т-схема замещения с общим током намагничивания 
диционной теории адекватных схем замещения, способных отобразить реальные физические процессы в аварийных ситуациях.

Предложенная 4Т-схема замещения чрезвычайно наглядна, однако степень ее приемлемости для описания изменений потоков в переходных процессах требует тщательной проверки путем сравнения с результатами расчета по другим схемным моделям автотрансформатора, которые могут быть получены на

\section{Приведенная 4Т-схема замещения идеализированного повышающего автотрансформатора}

Левая и правая части неприведенной схемы замещения (рис. 3, б) разделены конвертором в виде идеального трансформатора $\left(n_{b}^{a}=w_{a} / w_{b}\right)$. Эту схему можно преобразовать, перенеся по очереди элементы правой части $\left(3 L_{b} / 2,-L_{b} / 2\right.$ и $\left.Z_{\mathrm{H}}\right)$ через конвертор в левую часть. Общие принципы переноса элементов схем через 2×2-полюсники сформулированы в [17]. В нашем случае достаточно знать следующее правило:

при переносе продольной индуктивности или сопротивления через идеальный трансформатор справа налево они умножаются на $n_{b}^{a 2}$; при этом напряжения на них «увеличиваются» в $n_{b}^{a}$ раз при «уменьшении» тока во столько же раз (мощность, выделяемая на этих элементах после переноса остается без изменений); место, где располагались продольные элементы закорачивается.

На рис. 10, $а$ показаны первые два этапа реализации этого правила для элементов схемы, изображенной на рис. 3,6 .

Сначала перенесена индуктивность $3 L_{b} / 2$, которая после умножения на $n_{b}^{a 2}$ превратилась в новую индуктивность

$$
n_{b}^{a 2} \frac{3}{2} L_{b} \rightarrow \frac{3}{2} L_{b}^{\prime}
$$

с напряжением на ней

$$
j \omega w_{b} \dot{\Phi}_{b} n_{b}^{a}=j \omega\left(w_{b} n_{b}^{a}\right) \dot{\Phi}_{b} \rightarrow j \omega w_{a} \dot{\Phi}_{b},
$$

при этом поперечное напряжение за ней превратилось в следующее:

$$
j \omega w_{b} \dot{\Phi}_{\text {бок }} n_{b}^{a}=j \omega\left(w_{b} n_{b}^{a}\right) \dot{\Phi}_{\text {бок }} \rightarrow j \omega w_{a} \dot{\Phi}_{\text {бок }} .
$$

На втором этапе перенесена индуктивность $-L_{b} / 2$, в результаты чего образовалась схема на рис. 10, $a$. Здесь же удобства ради для осуществления следующего этапа сопротивление нагрузки $Z_{\mathrm{H}}$ пред- основе 2Т-схем замещения трансформаторов [19] или по еще более точным магнитоэлектрическим схемам их замещения [20]. В целом достоверность разработанной теории подтверждается практическим совпадением экспериментальных измерений магнитных потоков в стали опытного образца автотрансформатора с результатами их расчетов на основе 4Т-образной схемы замещения в режиме установившегося К3 автотрансформатора.

\section{ПРИЛОЖЕНИЕ}

ставлено в виде продольной ветви. Пунктиром показано напряжение за нагрузкой, совпадающее с входным напряжением автотрансформатора $\dot{U}_{a}$.

Третий этап после переноса $Z_{\mathrm{H}}$ через конвертор представлен на рис. 10, б. В результате заданное напряжение $\dot{U}_{a}$ оказывается приложенным непосредственно к правым зажимам идеального трансформатора, что, очевидно, делает известным и напряжение слева от него, которое схема воспринимает как ЭДС, равную $n_{b}^{a} \dot{U}_{a}$. Поэтому схему на рис. $10, \sigma$ можно также изобразить с дополнительной ЭДС справа, как показано на схеме рис. 10, в, в которой зависимый источник тока $\dot{I}_{b}$, имитирующий правую ветвь идеального трансформатора, перемещен в начало схемы.

На схеме рис. 10, в наглядно представлены все те же электрические и магнитные величины, что и на исходной схеме рис. 3 , б с четырьмя магнитными потоками в стали. Поэтому она может быть названа приведенной 4Т-образной схемой замещения идеализированного повышающего автотрансформатора. Мощность, потребляемая в нагрузке $n_{b}^{a 2} Z_{\mathrm{H}}$, равна мощности, потребляемой нагрузкой $Z_{\mathrm{H}}$ в исходной цепи (рис. 3, б, в).

Для расчета тока $\dot{I}_{a}$ схему на рис. 10, в можно упростить, отбросив зависимый источник тока (рис. 11). Далее тривиальным объединением всех индуктивностей, а также суммированием ЭДС

$$
\dot{U}_{a}+n_{b}^{a} \dot{U}_{a}=\left(1+n_{b}^{a}\right) \dot{U}_{a}=\frac{\dot{U}_{a}}{n_{b}}
$$

получаем свернутую схему замещения (см. рис. 4, б), в которой также можно увидеть магнитные потоки $\dot{\Phi}_{я}^{a}$ и $\dot{\Phi}_{я}^{b}$. Для этого в ней следует провести поперечные стрелки от центральной индуктивности $L_{\delta}^{a}$. Очевидно, левая стрелка будет соответствовать напряжению $j \omega w_{a} \dot{\Phi}_{я}^{a}+n_{b}^{a} \dot{U}_{a}$, аправая-напряжению $j \omega w_{a} \dot{\Phi}_{я}^{b}+n_{b}^{a} \dot{U}_{a}$.

В отличие от схемы рис. 4, б схема замещения на рис. 11 «физичнее». Во-первых, тем, что в ней отображаются все магнитные потоки. А во-вторых, 

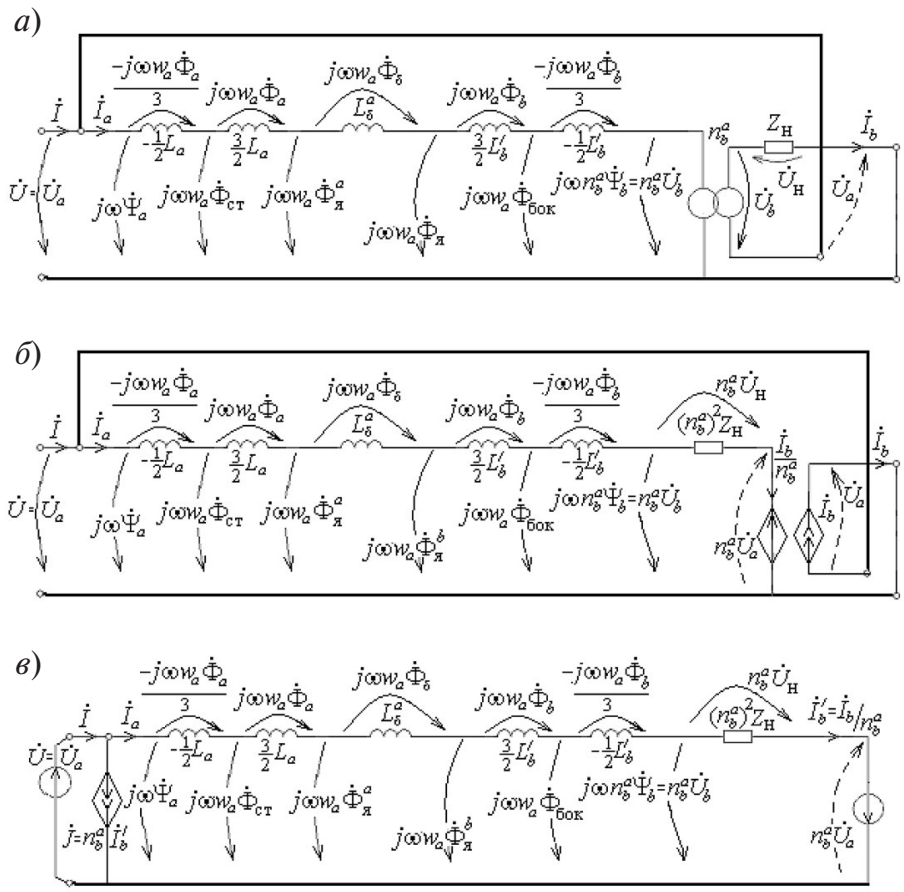

Рис. 10. Схема замещения идеализированного автотрансформатора после переноса через конвертор справа налево индуктивных элементов $(a)$ и сопротивления нагрузки (б), а также замены ИНУН независимой ЭДС (в)

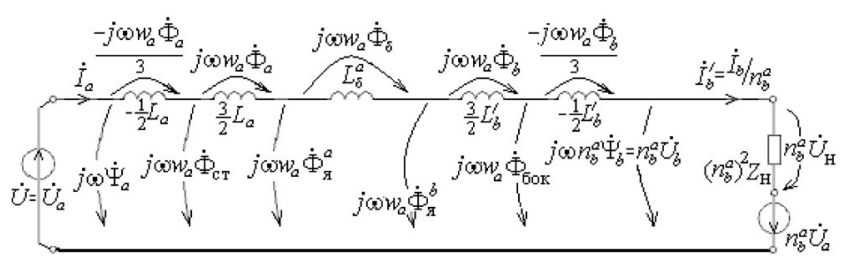

Рис. 11. Приведенная развернутая схема автотрансформатора относительно тока $\dot{I}_{a}$

прямой демонстрацией преимущества автотрансформатора, состоящего в передаче мощности в нагрузку двумя путями, электромагнитным и электрическим. Электромагнитная (или трансформаторная) мощность передается от левого источника ЭДС с напряжением $\dot{U}=\dot{U}_{a}$. Она равна

$$
\dot{S}_{\text {эм }}=\dot{U}_{a} \stackrel{*}{I}_{a}=\dot{U} I_{a}^{*}
$$

а электрическая - от правого источника ЭДС с напряжением $n_{b}^{a} \dot{U}_{a}$. Она равна

$$
\dot{S}_{\ni}=\left(n_{b}^{a} \dot{U}_{a}\right) \stackrel{*}{I}_{a}=\dot{U}\left(n_{b}^{a} \stackrel{*}{I}_{a}\right)=\dot{U} \dot{I}_{b}^{*} .
$$

В сумме они образуют мощность, подводимую к входным зажимам автотрансформатора:

$$
\dot{S}=\dot{S}_{\text {эм }}+\dot{S}_{\ni}=\dot{U} \dot{I}_{a}^{*}+\dot{U} I_{b}^{*}=\dot{U}\left(\dot{I}_{a}^{*}+\stackrel{*}{I}_{b}\right)=\dot{U} \dot{I}^{*} .
$$

Из схемы на рис. 11 следует, что мощность, потребляемая нагрузкой, равна

$$
\dot{S}_{\mathrm{H}}=\left(n_{b}^{a} \dot{U}_{b}-n_{b}^{a} \dot{U}_{a}\right) \stackrel{I}{a}_{a}^{*}=\left(\dot{U}_{b}-\dot{U}_{a}\right)\left(n_{b}^{a} \stackrel{I}{I}_{a}^{*}\right)=\dot{U}_{\mathrm{H}} \stackrel{*}{I}_{b} .
$$

Если положить все индуктивности равными нулю, то $\dot{S}=\dot{S}_{\text {н }}$, чем и обусловлено название $\dot{S}$ как проходной мощности.

Преобразуем схему (рис. 10, в) с помощью леммы, задавшись коэффициентами $\eta=n_{b}$ и $\xi=n_{b}^{2}$. В этом случае согласно лемме токи изменятся в $\eta / \xi=1 / n_{b}$ раз:

$$
\begin{gathered}
\dot{I} \rightarrow \frac{\dot{I}}{n_{b}} ; \quad \dot{J} \rightarrow \frac{\dot{J}}{n_{b}}=\frac{\dot{I}_{b}}{n_{b}} ; \quad \dot{I}_{a} \rightarrow \frac{\dot{I}_{a}}{n_{b}}=\dot{I} ; \\
\dot{I}_{b}^{\prime} \rightarrow \frac{\dot{I}_{b}^{\prime}}{n_{b}}=\frac{\dot{I}_{b}}{n_{b}^{a} n_{b}}=\frac{\dot{I}_{b}}{n_{a}} .
\end{gathered}
$$



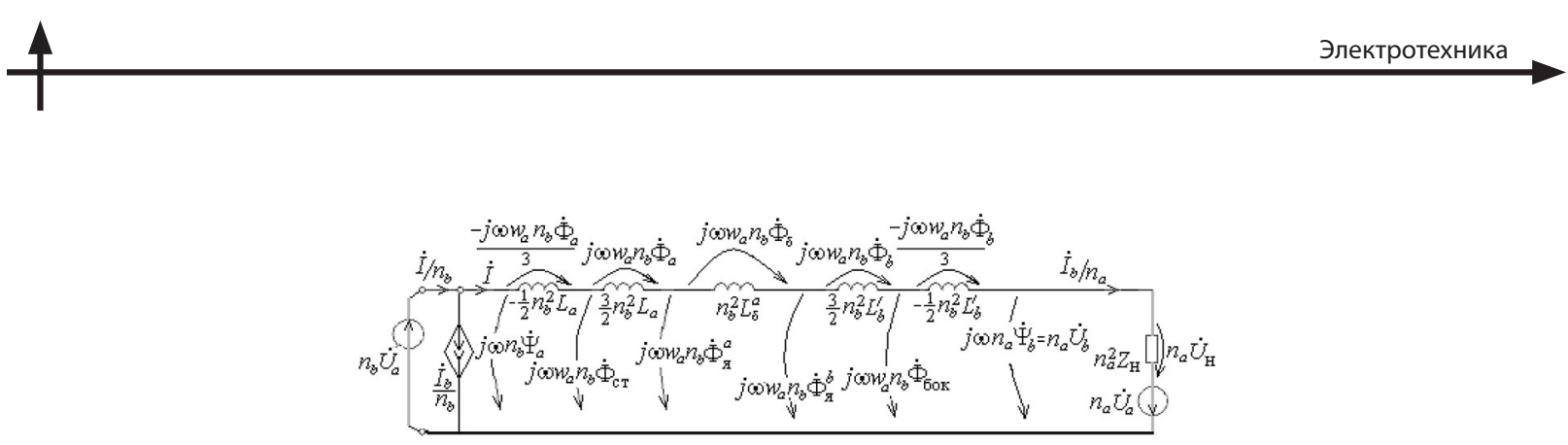

Рис. 12. 4Т-образная схема замещения идеального автотрансформатора, родственно-эквивалентная схеме на рис. 11

И родственно эквивалентная схема примет вид (рис. 12). В ней все индуктивности увеличены в $\xi=n_{b}^{2}$ раз. Аналогичным образом изменено сопротивление нагрузки:

$$
n_{b}^{a 2} Z_{\mathrm{H}} \rightarrow n_{b}^{2} n_{b}^{a 2} Z_{\mathrm{H}}=\left(n_{b} n_{b}^{a}\right)^{2} Z_{\mathrm{H}}=n_{a}^{2} Z_{\mathrm{H}} .
$$

Все напряжения увеличены в $\eta=n_{b}$ раз. В частности, для напряжения на нагрузке имеем

$$
n_{b}^{a} \dot{U}_{\mathrm{H}} \rightarrow n_{b} n_{b}^{a} \dot{U}_{\mathrm{H}}=\left(n_{b} n_{b}^{a}\right) \dot{U}_{\mathrm{H}}=n_{a} \dot{U}_{\mathrm{H}} .
$$

Аналогично для напряжения на правом источнике ЭДС запишем

$$
n_{b}^{a} \dot{U}_{a} \rightarrow n_{b} n_{b}^{a} \dot{U}_{a}=\left(n_{b} n_{b}^{a}\right) \dot{U}_{a}=n_{a} \dot{U}_{a}
$$

Точно также преобразовано поперечное напряжение:

$j \omega n_{a}^{b} \dot{\Psi}_{b} \rightarrow j \omega n_{b} n_{b}^{a} \dot{\Psi}_{b}=j \omega\left(n_{b} n_{b}^{a}\right) \dot{\Psi}_{b}=j \omega n_{a} \dot{\Psi}_{b}=n_{a} \dot{U}_{b}$.

Для определения тока $\dot{I}$ схему можно упростить, отбросив источник тока и объединив в ней источники ЭДС:

$$
n_{b} \dot{U}_{a}+n_{a} \dot{U}_{a}=\left(n_{b}+n_{a}\right) \dot{U}_{a}=\dot{U}_{a} .
$$

После чего схема на рис. 12 почти совпадет со схемой на рис. 7, б. Для полного совпадения следует при записи напряжений на индуктивностях учесть, что

$$
w_{a} n_{b}=w_{b} n_{a} .
$$

Bсе схемы на рис. 10-12 эквивалентны по мощности, потребляемой в нагрузке.

\section{СПИСОК ЛИТЕРАТУРЫ}

1. Вольдек А.И. Электрические машины. Л.: Энергия, $1974.840 \mathrm{c}$.

2. Иванов-Смоленский А.В. Электрические машины. М.: Энергия, 928 с.

3. Петров Г.Н. Электрические машины. Ч. 1: Трансформаторы. М.: Энергия, 1974. 240 стр.

4. Важнов А.И. Электрические машины. Л.: Энергия, $1968.708 \mathrm{c.}$

5. Сергеенков Б.Н., Киселев В.М., Акимова Н.А. Электрические машины: Трансформаторы / Под ред. И.П. Копылова. М.: Высшая школа, 1989. 352 с.

6. Лейтес Л.В., Пинцов А.М. Схемы замещения многообмоточных трансформаторов. М.: Энергия, 1974. $192 \mathrm{c}$.

7. Васютинский С.Б. Вопросы теории и расчета трансформаторов. Л.: Энергия, 1970. 432 с.

8. Конов Ю.С. Насыщение магнитопроводов трансформаторов и автотрансформаторов при коротком замыкании // Электротехническая промышленность. Сер.: Аппараты высокого напряжения, трансформаторы, силовые конденсаторы. 1976. Вып. 11 (67).

9. Григоров И.Б. Потоки в магнитопроводах мощных силовых трансформаторов и автотрансформаторов с учетом потока рассеяния // Электротехника. 1975. №4. C. 20-25.

10. Boyajian A. Resolution of transformer reactances into primary and secondary reactances // AIEE Trans. Jun. 1925. P. 805-810.
11. Cherry E.C. The duality between interlinkend electric and magnetic circuits and the formulation of transformer equivalent circuits // Proceedings of the Physical Society. Vol. (B) 62, Feb. 1949. P. 101-111.

12. Blume L.F., Boyajian A., Gamilly G., Lenox T.C. Minnec S. Montsinger M.V. Transformer Engineering: A treatise on the Theory, Operation and Application of Transformer. New York: Wiley, 1951. 239 c.

13. Александров Г.Н., Шакиров М.А. Трансформаторы и реакторы: новые идеи и принципы. СПб.: Изд-во Политехн. ун-та, 2006. 204 с.

14. Шакиров М.А. Вектор Пойнтинга и новая теория трансформаторов. Часть $1 / /$ Электричество. 2014. № 9. C. 52-59.

15. Шакиров М.А. Вектор Пойнтинга и новая теория трансформаторов. Часть 2 // Электричество. 2014. № 10. C. 53-65.

16. Практикум по ТОЭ / Под ред. М.А.Шакирова. СПб.: Из-во СПбГПУ, 2006, 252 с.

17. Шакиров М.А. Перенос элементов электрической цепи через 2х2-полюсники // Электричество, 2006, № 11. С. 45-53.

18. Шакиров М.А., Андрущук В.В., Дуань Лиюн. Аномальные магнитные потоки в двухобмоточном трансформаторе при коротком замыкании // Электричество. 2010. № 3. С. 55-63.

19. Шакиров М.А. 2Т-образные схемы замещения трансформаторов // Электричество. 2010. № 5. С. 19-36. 
20. Шакиров М.А. Анализ неравномерности распределения магнитных нагрузок и потерь в трансформаторах на основе магнитоэлектрических схем замещения // Электричество. 2005. № 11. С. 15-27.
21. Левицкая Е.И., Лурье А.И., Панибратец А.Н. Проблема электродинамической стойкости трансформаторов при коротких замыканиях // Электротехника. 2001. №9. С. 47-54.

\section{REFERENCES}

1. Voldek A.I. Elektricheskiye mashiny [Electrical machinery.] L.: Energiya, 1974. 840 s. (rus.)

2. Ivanov-Smolenskiy A.V. Elektricheskiye mashiny. [Electrical machinery.] M.: Energiya, 1980. 928 str. (rus.)

3. Petrov G.N. Elektricheskiye mashiny. Ch1. Transformatory. [Electrical machinery. Part. 1. Transformatory.] M.: Energiya, 1974. 240 s. (rus.)

4. Vazhnov A.I. Elektricheskiye mashiny. [Electrical machinery]. L.: Energiya, 1968. 708 s. (rus.)

5. Sergeyenkov B.N., Kiselev V.M., Akimova N.A. Elektricheskiye mashiny: Transformatory [Electrical Machines: Transformers.] Pod red. I.P.Kopylova. M.: Vyssh. shk., 1989, 352 s. (rus.)

6. Leytes L.V., Pintsov A.M. Skhemy zameshcheniya mnogoobmotochnykh transformatorov. [Equivalent circuit multiwinding transformers.] M.: Energiya, 1974, 192 s. (rus.)

7. Vasyutinskiy S.B. Voprosy teorii i rascheta transformatorov [Theory and calculation of transformers.] L.: Energiya, 1970. 432 s. (rus.)

8. Konov Yu.S. Nasyshcheniye magnitoprovodov transformatorov i avtotransformatorov pri korotkom zamykanii. Saturation of magnetic transformers and autotransformers short-circuit. Elektrotekhnicheskaya promyshlennost. Ser.: Apparaty vysokogo napryazheniya, transformatory, silovyye kondensatory [Electrical industry. Ser.: Washer voltage transformers, power capacitors]. 1976. Vyp.11 (67). (rus.)

9. Grigorov I.B. Potoki v magnitoprovodakh moshchnykh silovykh transformatorov i avtotransformatorov s uchetom potoka rasseyaniya. [Flows in the magnetic powerful power transformers and autotransformers with the leakage flux. Elektrotekhnika. 1975. №4. S. 20-25. (rus.)

10. Boyajian A. Resolution of transformer reactances into primary and secondary reactances AIEE Trans. Jun. 1925. P. 805-810.

11. Cherry E.C. The duality between interlinkend electric and magnetic circuits and the formulation of transformer equivalent circuits. Proceedings of the Physical Society. Feb. 1949. Vol. (B) 62. P. 101-111.
12. Blume L.F., Boyajian A., Gamilly G., Lenox T.C. Minnec S. Montsinger M.V. Transformer Engineering: A treatise on the Theory, Operation and Application of Transformer. New York: Wiley, 1951, 239 s.

13. Aleksandrov G.N., Shakirov M.A. Transformatory i reaktory: novyye idei i printsipy [Transformatory and reactors, new ideas and principles]. SPb.: Izd-vo Politekhn. un-ta, 2006. 204 s. (rus.)

14. Shakirov M.A. Vektor Poyntinga i novaya teoriya transformatorov. Chast 1. [Poynting vector and the new theory of transformers. Part 1. Elektrichestvo. 2014. № 9. S. 52-59. (rus.)

15. Shakirov M.A. Vektor Poyntinga i novaya teoriya transformatorov. Chast 2. [Poynting vector and the new theory of transformers. Part 2]. Elektrichestvo. 2014. № 10. S. 53-65. (rus.)

16. Praktikum po TOE [Workshop on SOC] / Pod red. M.A. Shakirova. SPb.: Izd-vo SPbGPU, 2006. 252 s. (rus.)

17. Shakirov M.A. Perenos elementov elektricheskoy tsepi cherez 2kh2-polyusniki [Transferring the circuit elements through the 2x2-poles]. Elektrichestvo. 2006. № 11. S. 45-53. (rus.)

18. Shakirov M.A., Andrushchuk V.V., Duan Liyun. Anomalnyye magnitnyye potoki v dvukhobmotochnom transformatore pri korotkom zamykanii[Anomalous magnetic fluxes in the two-winding transformer short-circuit]. Elektrichestvo. 2010. № 3. S. 55-63. (rus.)

19. Shakirov M.A. 2T-obraznyye skhemy zameshcheniya transformatorov [2T-shaped equivalent circuit transformers.]. Elektrichestvo. 2010. № 5. S. 19-36. (rus.)

20. Shakirov M.A. Analiz neravnomernosti raspredeleniya magnitnykh nagruzok i poter $\mathrm{v}$ transformatorakh na osnove magnitoelektricheskikh skhem zameshcheniya [Analysis of the uneven distribution of magnetic loads and losses in transformers based on magnetoelectric equivalent circuits]. Elektrichestvo. 2005. № 11. S. 15-27. (rus.)

21. Levitskaya Ye.I., Lurye A.I., Panibratets A.N. Problema elektrodinamicheskoy stoykosti transformatorov pri \korotkikh zamykaniyakh [Problem withstand transformers during short circuits.]. Elektrotekhnika. 2001. №9. S. 47-54. (rus.) 


\section{СВЕДЕНИЯ ОБ АВТОРЕ}

ШАКИРОВ Мансур Акмелович - доктор технических наук, Заслуженный работник высшей школы РФ, действительный член Нью-Йоркской Академии наук и Академии электротехнических наук РФ профессор кафедры теоретической электротехники и электромеханики Санкт-Петербургского политехнического университета Петра Великого. 195251, Россия, г. Санкт-Петербург, Политехническая ул., 29. E-mail: manshak@mail.ru

\section{AUTHOR}

SHAKIROV Mansur A. - Peter the Great St. Petersburg Polytechnic University. 29 Politechnicheskaya St., St. Petersburg, 195251, Russia. E-mail:manshak@mail.ru 